\title{
The effect of diversity maintenance on prediction in dynamic multi-objective optimization
}

\author{
Gan Ruan ${ }^{\mathrm{a}}$, Guo $\mathrm{Yu}^{\mathrm{c}, *}$, Jinhua Zheng ${ }^{\mathrm{a}, \mathrm{b}, *}$, Juan Zou ${ }^{\mathrm{a}, *}$, Shengxiang Yang ${ }^{\mathrm{d}, *}$ \\ ${ }^{a}$ Key Laboratory of Intelligent Computing and Information Processing (Ministry of \\ Education), Xiangtan University, Xiangtan, Hunan, 411105, China \\ ${ }^{b}$ Hunan Provincial Key Laboratory of Intelligent Information Processing and Application, \\ Hengyang Normal University, Hengyang, 421002, China \\ ${ }^{c}$ Computer Sciences, University of Surrey, Guildford, Surrey GU2 7XH, UK \\ ${ }^{d}$ School of Computer Science and Informatics, De Montfort University, Leicester LE1 9BH, \\ U.K.
}

\begin{abstract}
There are many dynamic multi-objective optimization problems (DMOPs) in real-life engineering applications whose objectives change over time. After an environmental change occurs, prediction strategies are commonly used in dynamic multi-objective optimization algorithms to find the new Pareto optimal set (POS). Being able to make more accurate prediction means the algorithm requires fewer computational resources to make the population approximate to the Pareto optimal front (POF). This paper proposes a hybrid diversity maintenance method to improve prediction accuracy. The method consists of three steps, which are implemented after an environmental change. The first step, based on the moving direction of the center points, uses the prediction to relocate a number of solutions close to the new Pareto front. On the basis of self-defined minimum and maximum points of the POS in this paper, the second step applies the gradual search to produce some well-distributed solutions in the decision space so as to compensate for the inaccuracy of the first step, simultaneously and further enhancing the convergence and diversity of the population. In the third step, some diverse individuals are randomly generated
\end{abstract}

\footnotetext{
* Corresponding author

Email addresses: ruangan199332@gmail.com (Gan Ruan), gy00059@surrey.ac.uk (Guo $\mathrm{Yu}$ ), jhzheng@xtu.edu.cn (Jinhua Zheng), zoujuan@xtu.edu.cn (Juan Zou), syang@dmu.ac.uk (Shengxiang Yang)
} 
within the region of next probable POS, which prompts the diversity of the population. Eventually the prediction becomes more accurate as the solutions with good convergence and diversity are selected after the non-dominated sort [1] on the combined solutions generated by the three steps. Compared with three other prediction methods on a series of test instances, our method is very competitive in convergence and diversity as well as the speed at which it responds to environmental changes.

Keywords:

Dynamic multi-objective optimization, Evolutionary algorithms, Diversity maintenance, Prediction

\section{Introduction}

Many multi-objective optimization problems in the real world have conflicting objectives, and their objective functions change over time [2]. These kinds of problems are dynamic multi-objective optimization problems (DMOPs). To

5 solve DMOPs, multi-objective evolutionary algorithms (MOEAs) are designed to find the Pareto optimal set (POS) before every new environmental change comes. Nevertheless, traditional MOEAs aim at finding the POS during the whole optimization process. When applied to solve DMOPs, traditional MOEAs [3] [4] [5] [6] have the limitation of a low speed of convergence and loss of di10 versity. Thus, the key factor in solving DMOPs is to find the POS quickly and efficiently in a limited time period. Under this circumstance, static MOEAs are improved to adapt to the environmental changes [7] [8] [9].

In recent years, many kinds of dynamic multi-objective evolutionary algorithms (DMOEAs) have been proposed to solve DMOPs, such as artificial im15 mune algorithms [10] [11], co-evolutionary algorithms [12], particle swarm optimization algorithms [13] [14] [15], memetic computing [16], and other methods [10] [14] [17] [18] [19] [20] [21] [22] [23]. Additionally, some strategies have been adopted into the static MOEAs to deal with DMOPs like reinitializing population [13] [24] [25] [26] [27] [28], hyper-mutation [29], dynamic migration [21], 
and multiple population [12] [27].

Particularly, some researchers have put forward strategies based on prediction [14] [30] [31] [32] and memory [33] to solve DMOPs. The prediction strategy can guide the population to evolve towards the POF after each environmental change, making algorithms respond quickly to the environmental change. For example, Hatzakis and Wallace proposed a feed-forward prediction strategy (FPS) [34]. Zhou and Jin et al. proposed a population prediction strategy (PPS) [35]. Their prediction models are based on the autoregressive model. Another prediction model called the Kalman Filter model was put forward by Muruganantham et al. [36]. These methods [34] [35] [36], which are based on so prediction, achieve good results in solving DMOPs to some extent. However, there are also some defects. For instance, it is difficult for the FPS to solve DMOPs which have nonlinear correlation between decision variables. Additionally, the convergence speed of PPS is slow in the early period of environmental changes due to the lack of historical information accumulation. The prediction of 35 FPS and PPS is built on the historical information, which may mislead the population in the later prediction during optimization. Memory strategies mainly reuse previous optimal solutions stored in a memory to quickly respond to the new environmental change. These strategies perform well on periodic problems. But in the earlier period of environmental changes or for the non-periodic prob40 lems, memory strategies are less effective for enhancing convergence [12] [37] [38].

The integration of the prediction and memory strategies is a novel trend in solving DMOPs [27]. The prediction strategy makes the algorithm quickly respond to environmental change and helps to find the new POS. Meanwhile, the memory strategy has the ability to deal with periodic problems and decrease the impact from inaccurate prediction. In recent years, some algorithms have been proposed to solve DMOPs using this hybrid strategy [39] [40] [41]. For example, Wang and Li put forward a multi-strategy ensemble evolutionary algorithm, called dMS-MOEA [39]. Koo et al. proposed a predictive gradient strategy 50 (EGS) [41]. Z. Peng et al. put forward a novel prediction and memory strategy 
(PMS) [42].

Particularly, the evolutionary gradient prediction in EGS is used to predict the new POS by recording the last predictive evolutionary gradients and the final two previous positions of the nondominated population center. As for the

${ }_{55}$ memory, EGS designs a memory pool to store past population centroids and the centroid variance, and then some individuals are produced by normal distribution sampling through what is stored in the memory pool. Being able to record the history of environmental changes, EGS can prompt the convergence and diversity to some extent. However, there are some shortcomings when solv6o ing problems with great environmental changes. Although EGS may decrease the impact that prediction causes using memory, it also loses diversity in the process of evolution.

According to [39] [40] [41] [42], predicting can benefit the convergence to some extent, but it may also misguide the population away from the true POF 65 in cases when the prediction is inaccurate. The memory strategy loses its advantage in the beginning of the optimization especially in dealing with non-periodic problems. Thus, to solve the problems in the stage of responding to environmental changes, this paper proposes a hybrid method which consists of three steps using: (1) prediction strategy; (2) gradual search strategy; (3) diversity 70 maintenance strategy. The prediction strategy is first used after an environmental change has been detected, making use of the moving direction of the center points to predict the POS, which can likely generate some solutions close to the new Pareto front. Thus, the first strategy used improves the convergence of the population. Second, the gradual search is applied, based on the 75 self-defined minimum and maximum points of POS, to produce a number of well-distributed solutions in the decision space. This strategy makes the search adequate, thereby enhancing the convergence and diversity of the population, and assisting the algorithm in responding to the environmental change quickly. In the final step, the diversity maintenance strategy is utilized to randomly so generate diverse individuals in the region of next probable POS, which can improve the population's diversity. Overall, the first strategy mainly plays the role 
of exploration, the other two strategies focus on exploitation. After the three steps, the non-dominated sort [1] is applied to sort the combination of newly generated solutions by the three steps. A group of solutions with good converge and diversity are selected as the responding population in accordance with the environmental change. In this way, more accurate prediction is obtained. Simultaneously, population with good convergence and diversity will also be gained. Importantly, the environmental changes are also responded to quickly during the optimization.

90

The rest of this paper is structured as follows. Section 2 describes the background information on DMOP. Section 3 presents the proposed algorithms. The test instances and evaluation metric are shown in Section 4. Section 5 presents the experimental results and analysis. Lastly, conclusions are summarized in Section 6 .

95 2. Background

\subsection{Dynamic multi-objective optimization}

In this paper, we consider the minimization problem and the dynamic multiobjective optimization problem (DMOP) [2], described as follows:

$$
\left\{\begin{array}{l}
\min \mathbf{F}(\mathbf{x}, t)=\left\{f_{1}(\mathbf{x}, t), f_{2}(\mathbf{x}, t), \ldots f_{M}(\mathbf{x}, t)\right\}, \\
\text { s.t. } g(\mathbf{x}, t) \leq 0, h(\mathbf{x}, t)=0
\end{array}\right.
$$

where $t$ is the time variable; $x=\left(x_{1}, x_{2}, \ldots, x_{n}\right)$ is the $\mathrm{n}$-dimension decision variables within the decision space $\Omega ; F=\left(f_{1}, f_{2}, \ldots, f_{n}\right)$ represents the set of $\mathrm{m}$ objectives to be minimized with respect to time $t$. And $g(\mathbf{x}, t) \leq 0$ and $h(\mathbf{x}, t)=0$ present the inequality and equality constraints.

Definition 1. Pareto Dominance: Supposing that $p$ and $q$ are any two individuals in the population, $p$ is said to dominate $q$, written as $f(p) \prec f(q)$ if $f_{i}(p) \leq f_{i}(q) \forall i \in 1,2, \ldots, m$ and $f_{j}(p)<f_{j}(q) \exists j \in 1,2, \ldots, m$. 
Definition 2. Pareto Optimal Set (POS): $x$ is the decision vector; $\Omega$ is the decision space; $F$ is the objective function. A solution is said to be nondominated if it is not dominated by any other solutions in $\Omega$. Thus, the POS [2] is the set of all nondominated solutions and can be defined mathematically as follows:

$$
P O S:=\left\{x \in \Omega \mid \neg \exists x^{*} \in \Omega, F\left(x^{*}\right) \prec F(x)\right\}
$$

Definition 3. Pareto Optimal Front (POF): $x$ is the decision vector; $\Omega$ is the decision space; $F$ is the objective function. Thus, the POF is the set of all nondominated solutions with respect to the objective space and can be defined mathematically as follows:

$$
P O F:=\{y=F(x) \mid x \in P O S\}
$$

According to the dynamic change of POS and POF, DMOPs were classified into four types by Farina et al. [2]:

- Type I: The POS changes with time but the POF is fixed.

- Type II: Both POS and POF change with time.

- Type III: The POS remains fixed, while the POF changes with time.

- Type $I V$ : Both POS and POF remain fixed.

In the real world, these four types of changes may occur at the same time when the environment changes. We mainly concentrate on the first three types of changes in dynamic multi-objective optimization (DMO). Fig. 1 illustrates a general flowchart of a dynamic multi-objective evolutionary algorithm. A dynamic multi-objective evolutionary algorithm generally has the following steps:

- Step 1. Initialize a population, set the initial parameters.

- Step 2. Change detection: if the environmental changes have been detected, the mechanism of change reaction would be evoked to respond to the changes; if not, static optimization algorithms would be continued. 


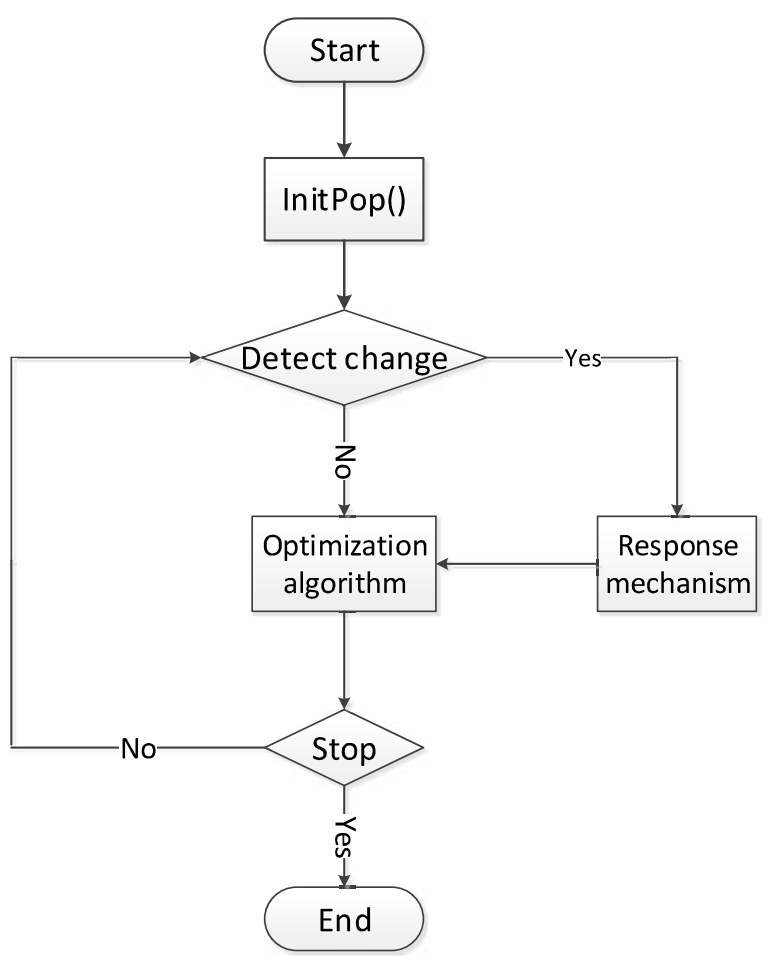

Figure 1: The flowchart of DMOEA

- Step 3. Optimization algorithm: RM-MEDA [43] [44], DE-NSGA-II are some frequently used algorithms in the DMO.

- Step 4. The mechanism of change reaction: the mechanism is designed to respond to environmental changes. There are several types of general mechanisms: re-initializing the population, parameter adjustment, dynamic migration, prediction, memory and so on.

- Step 5. Termination: if the stopping conditions are satisfied, then the optimization is terminated. 


\subsection{Related optimization algorithm $R M-M E D A$}

Estimation of distribution algorithms (EDAs) [45] are a new computing paradigm in evolutionary computation. Different from the process of evolutionary algorithm, EDAs explicitly extract globally statistical information from the selected solutions firstly, and then establish a posterior probability distribution model of promising solutions; new solutions are generated by sampling from the built probability model. On the basis of that, Zhang et al. [44] researched the distribution features of the solution set of a continuous multi-objective problem and proposed a regularity model-based multi-objective estimation of distribution algorithm referred to as RM-MEDA.

RM-MEDA can make full use of the global information provided by the current population to generate a new individual, without the need for local information directly used from population, which has a strong ability of heuristic optimization. Since RM-MEDA uses the regularity property of continuous MOPs, it is an excellent optimization algorithm for DMOPs and surpasses NSGA-II-DE in the DMOEA framework, and that has been verified in [35].

\section{The proposed algorithm}

In this section, two strategies are illustrated first. The first one is how to explore the POS by means of prediction when environmental change occurs. The second is how to exploit the well-converged and well-distributed solutions around the POS. Finally the main framework is presented.

\subsection{Exploration based on prediction}

The prediction strategy in this section is to find the new optimal solution set when the change is detected. Different from the complex prediction model used in FPS and PPS, this paper mainly makes use of the moving direction center points of the past two time's populations to predict the new location of POS. As the nondominated solutions are the elitist individuals of the current population and are able to show the position of the population, we choose the center point 
of nondominated solutions as the center of a population. Supposing that $C_{t}$ is the center of $P O S_{t}$, and $P O S_{t}$ is the nondominated solutions obtained at the end of time step $t$, then $C_{t}$ can be expressed by following formula:

$$
C_{t}=\frac{1}{\left|P O S_{t}\right|} \sum_{x_{t} \in P O S_{t}} x_{t}
$$

where $\left|P O S_{t}\right|$ is the number of the nondominated solutions in the POS at time $t$, and $x_{t}=\left(x_{t}^{1}, x_{t}^{2}, \ldots, x_{t}^{n}\right)$ is an individual at time $t$. Thus, the moving direction of center points referred to as $D_{t}$ at time $t$ can be defined as:

$$
D_{t}=C_{t}-C_{t-1}
$$

Then individuals at time $t+1$ would be generated by the predicted moving direction $D_{t}$ and the individuals of time $t$ according to the following formula:

$$
x_{t+1}=x_{t}+D_{t}
$$

where $x_{t}$ is the final obtained solution after the th environmental change, and the $x_{t+1}$ is the predicted solution of $(t+1)$ th change. The procedure of the prediction strategy is illustrated in Algorithm 1 in detail. Fig. 2 illustrates the process of prediction and generating solutions.

In Fig. 2, it can be seen that the center points are used to obtain the moving direction of the POS. The solutions denoted by black points are selected from the final population after the environmental change, which is also shown in the second step in Algorithm 1. The final predicted solution set is obtained in the third step in Algorithm 1. About Algorithm 1, $P_{t}$ is the population of time $t$, $N$ is the population size, and $P_{\text {prediction }}$ is the output population. In step 2 and step $3, r_{1} \in(0,1)$.

\subsection{Exploitation around the POS}

In Section. 3.1, the probable location of POS in the next time period has been predicted. The next step is to decrease the inaccuracy of the prediction as much as possible. Therefore, diversity and convergence are both taken into account. In this paper, we are proposing two strategies such as gradual search 


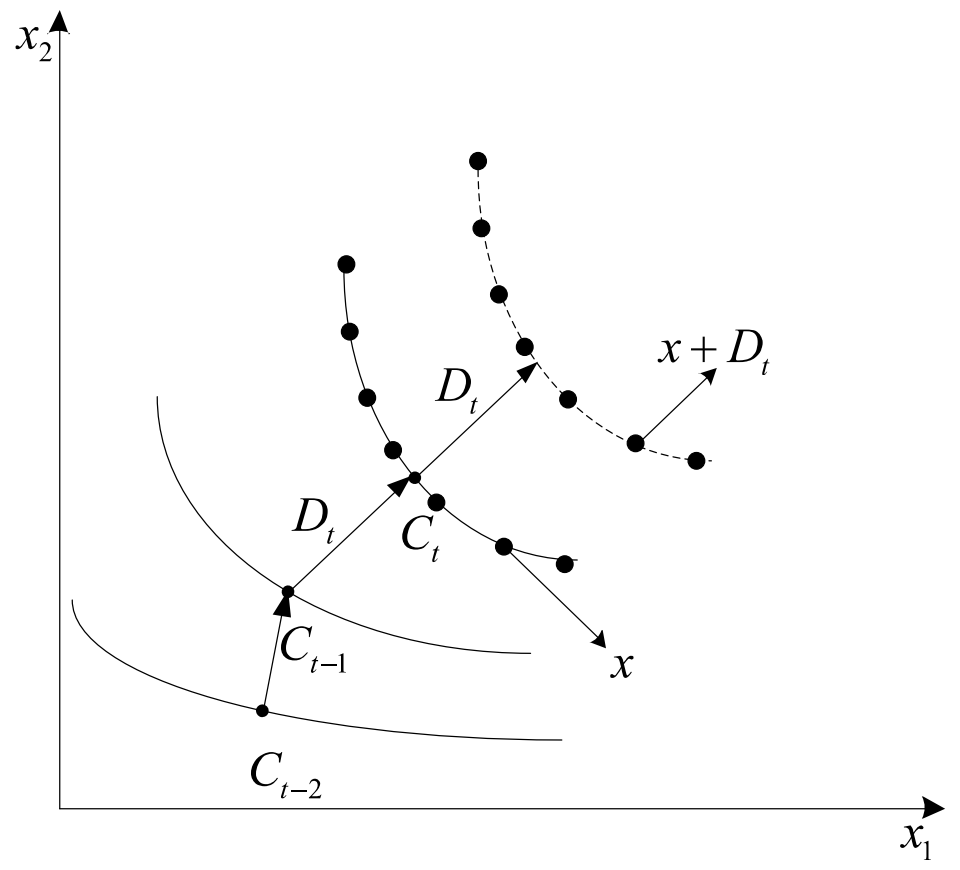

Figure 2: Exploration of the population

strategy and random diversity maintenance strategy. The first one puts more emphasis on convergence, and the second one concentrates more on diversity.

\subsubsection{Gradual search strategy}

The gradual search strategy is used to find well-converged and well-distributed solutions around the predicted POS. This strategy is different with the prediction shown in Section. 3.1, where the exploration is to predict the POS straightforwardly. If the POS of the problem is complex, then the prediction may be inaccurate. But the gradual search strategy can decrease the effect of inaccuracy because it will generate more solutions between the current POS and predicted POS. The solutions will benefit the convergence and diversity of the population 
Algorithm 1 Exploration based on prediction

Require: $P_{t}, P O S_{t}, N, C_{t-1}$;

Ensure: $P_{\text {prediction; }}$

1: Calculate the center $C_{t}$ of $P O S_{t}$ and $D_{t}$ according to formulas (4) and (5).

2: Calculate the crowding-distance of each individual of $P_{t}$ in the objective space, select $r 1 \times N$ individuals from $P_{t}$ with the largest crowding-distance.

3: Generate $r 1 \times N$ individuals according to formula (6) and boundary check them.

4: Put them into $P_{\text {prediction }}$ and return $P_{\text {prediction }}$.

in the optimization.

This strategy mainly makes use of the minimum point $\left(l^{\circ o w_{t}}\right)$ and the maximum point $\left(h i g h_{t}\right)$ of the first layer of non-dominated solutions after the nondominated sorting [1]. The definition of the minimum point and the maximum point are defined in Eq. (7). In this strategy, the moving direction is applied to determine the searching region. The moving directions of the minimum point and maximum point between two environmental changes are denoted by low $D_{t}$ and high $D_{t}$ respectively.

The $l o w_{t}$ and $h i g h_{t}$ are the minimum point and maximum point respectively at time period $t$ and the definition of each is as follows:

$$
\left\{\begin{array}{l}
\operatorname{low}_{t}=\left(\operatorname{low}_{t}^{1}, \ldots, \text { low }_{t}^{i}, \ldots, \text { low }_{t}^{n}\right) \\
\text { high }_{t}=\left(\text { high }_{t}^{1}, \ldots, \text { high }_{t}^{i}, \ldots, \text { high }_{t}^{n}\right)
\end{array} \quad, i=1,2 \ldots n\right.
$$

where $n$ is the dimension of the decision space. Then $i$ th element of the minimum point and maximum point, referred to as $l o w_{t}^{i}$ and $h i g h_{t}^{i}$, is defined as:

$$
\left\{\begin{array}{l}
\operatorname{low}_{t}^{i}=\min _{j=1}^{K}\left(x_{t}^{i j}\right) \\
h i g h_{t}^{i}=\max _{j=1}^{K}\left(x_{t}^{i j}\right)
\end{array} \quad x_{t}^{i j}=\left(x_{t}^{i 1} \ldots x_{t}^{i j} \ldots x_{t}^{i K}\right) \in \text { POS }_{t}\right.
$$

where $K$ is the number of the solutions in the first layer of non-dominated solu- 
tions after the non-dominated sorting. The moving directions of the minimum point and maximum point at time $\mathrm{t}$, referred to as $l o w D_{t}$ and $h i g h D_{t}$, can be formulated by the following:

$$
\left\{\begin{array}{l}
\operatorname{low} D_{t}=\left(\operatorname{low} D_{t}^{1}, \ldots, \text { low } D_{t}^{i}, \ldots, \text { low } D_{t}^{n}\right) \\
\text { high } D_{t}=\left(h i g h D_{t}^{1}, \ldots, \text { high } D_{t}^{i}, \ldots, \text { high } D_{t}^{n}\right)
\end{array} \quad, i=1,2 \ldots n\right.
$$
direction of the minimum point and maximum point referred to as $l o w D_{t}^{i}$ and $h i g h D_{t}^{i}$ is defined as:

$$
\left\{\begin{array}{l}
\operatorname{low} D_{t}^{i}=l o w_{t}^{i}-l o w_{t-1}^{i} \\
\operatorname{high}_{t}^{i}=h i g h_{t}^{i}-h_{i g h}^{i}
\end{array}\right.
$$

Then the moving direction is selected by $\max \left(\left|\operatorname{low} D_{t}\right|, \mid\right.$ high $\left.D_{t} \mid\right),\left|\operatorname{low} D_{t}\right|$ and $\left|h i g h D_{t}\right|$ are the length of the moving directions. Supposing that $\left|h i g h D_{t}\right|=$ Algorithm 2 Gradual search strategy Require: $P_{t}, P O S_{t}, P O S_{t-1}, N$;

Ensure: PGraSearch;

1: Calculate the minimum point and maximum point of $t-1$ and $t$ according to Eq. (7), then calculate the moving direction of the minimum point and maximum point $l o w D_{t}$ and $h i g h D_{t}$ according to Eq. (10).

2: Supposing that the length of high $D_{t}$ is larger than that of $l o w D_{t}$, then high $D_{t}$ is equally divided into $N / m$ parts.

3: Calculate the crowding-distance of each individual of $\mathrm{POS}_{t}$ in the decision space and select $m$ individuals from $P O S_{t}$ whose crowding-distance is the largest in turns.

4: Generate $N$ individuals according to formula (11) and boundary check them.

5: Put them into $P_{\text {GraSearch }}$ and return $P_{\text {GraSearch }}$. $\max \left(\left|\operatorname{low} D_{t}\right|,\left|h i g h D_{t}\right|\right)$, then the main steps of the gradual search strategy are described in Algorithm 2. Firstly, it calculates the minimum and maximum 
points and the moving direction in terms of Eqs. (7)(8)(9)(10). In step 2, high $D_{t}$ is equally divided into $N / m$ parts. Then in step 3 , the crowding-distance of each individual of the current population in the decision space is calculated. Lastly, step 4 generates $\mathrm{N}$ solutions by means of Eq. (11).

$$
\left\{\begin{array}{l}
y_{i * j}=x_{t}^{i}+\frac{h i g h D_{t}}{N / m} * j+\text { random }(-r, r) \\
i=0,1, \ldots, m ; j=0,1, \ldots, N / m
\end{array}\right.
$$

where $r$ is a small radius. $y_{i * j}$ is the $i * j$ th solution. $x_{t}^{i}$ is the $i$ th solution in the previous POS in the $t$ th environmental change. $P_{t}$ is the population of time $t$, and $N$ is the number of the individuals in the population, and $P_{\text {GraSearch }}$ is the output population.

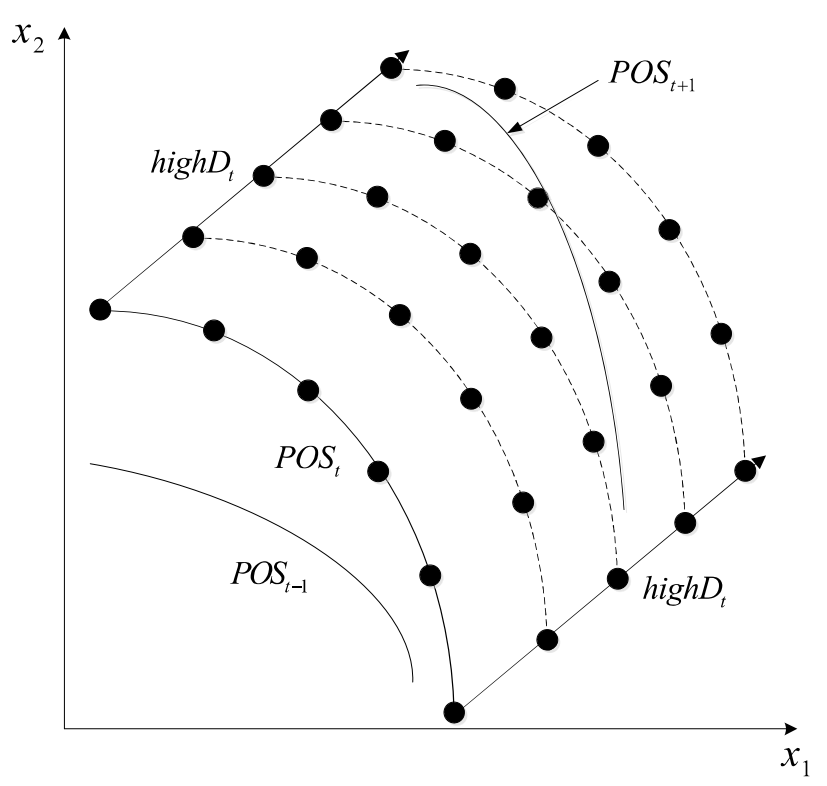

Figure 3: Gradual search on the decision space

Fig. 3 gives the illustration of how to gradually search the decision space. It mainly makes use of $P O S_{t}$ and $h i g h D_{t}$ to gradually generate solutions. 


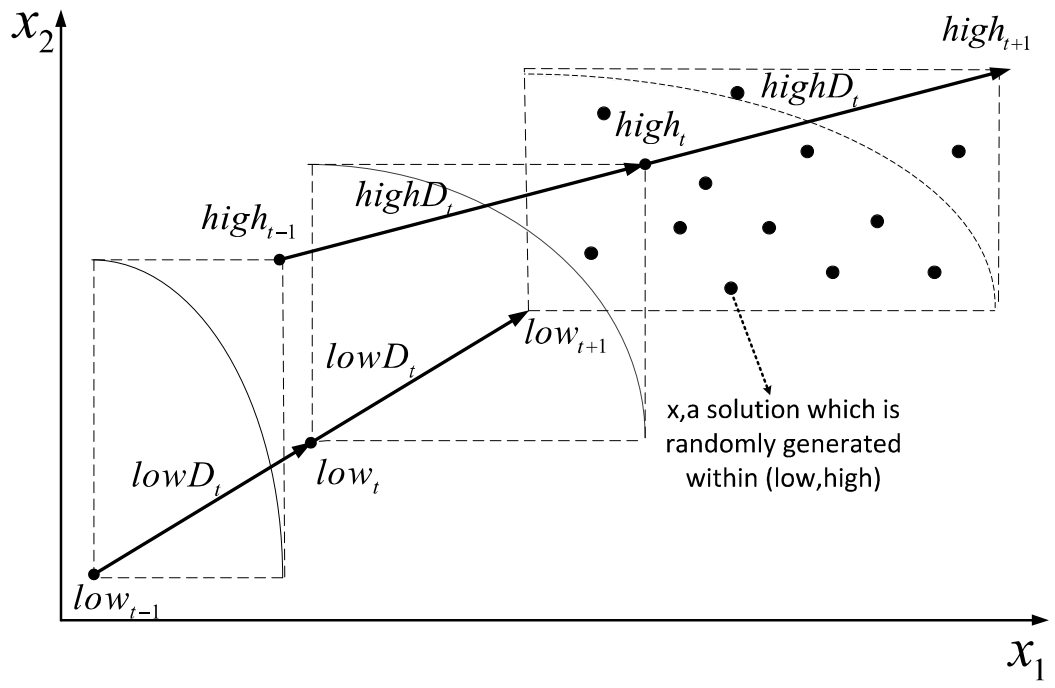

Figure 4: Random generate individuals in promising $P O S_{t+1}$

\subsubsection{Random diversity maintenance strategy}

The first two strategies contribute to the convergence of the population. In order to enhance the diversity of the population, the random diversity maintenance strategy is proposed. In Fig. 4, an example is presented to illustrate the idea of the random diversity maintenance strategy. low $w_{t+1}$ and $h i g h_{t+1}$, obtained by Eq. (12), are applied to determine the searching decision space. They can be obtained by Eq. (12). Solutions in the determined decision space are generated as shown in Eq. (13).

$$
\left\{\begin{array}{l}
\operatorname{low}_{t+1}=\operatorname{low}_{t}+\operatorname{low} D_{t} \\
\operatorname{high}_{t+1}=\operatorname{high}_{t}+\operatorname{high} D_{t}
\end{array}\right.
$$

where low $_{t}$ and high $h_{t}$ can be obtained by Eq. (7) and low $D_{t}$ and highDt can be obtained by Eq. (9). Thus, the individuals are generated as follows:

$$
x_{t+1}=\operatorname{random}\left(\operatorname{low}_{t+1}, \text { high }_{t+1}\right)
$$


where $\operatorname{random}(\mathrm{a}, \mathrm{b})$ is a random function and returns a random value between $\mathrm{a}$ and $\mathrm{b}$.

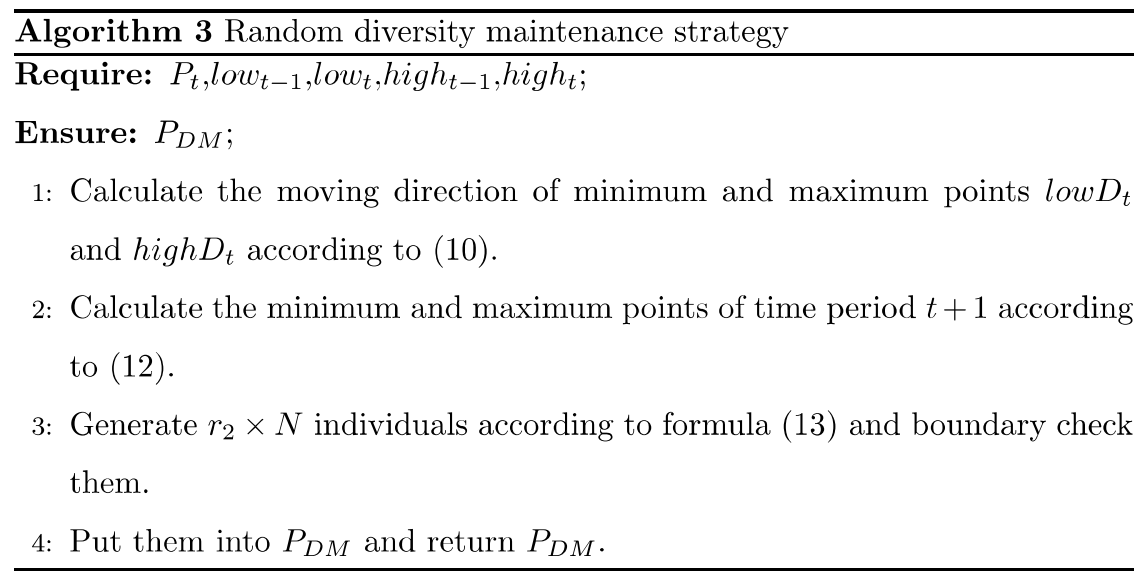

The details of the diversity maintenance strategy are given in Algorithm 3. $P_{t}$ is the final population after the $t$-th environmental change, and $P_{D M}$ is the output population. Especially, in step 3, $r_{2} \in(0,1)$.

\subsection{Overall framework of the proposed strategy}

These three strategies (prediction, gradual search, random diversity maintenance) are added into the response mechanism of the general DMOEA shown in Fig. 1. The framework of the algorithm is denoted by DMS-MOEA, and is presented in Algorithm 4.

The initialization of the algorithm is shown in step 1 . In step $2, P_{\text {duplicapte }}$ is an extra archive to save the duplicated population. Step 3 is the exploration, which is used to predict the POS to respond to the environmental change, and the $P_{\text {duplicapte }}$ is the input population of Algorithm 1. Similarly, step 4 and step 5 are the gradual search strategy and random diversity maintenance strategy, respectively, and the combination of step 4 and step 5 plays the role of exploitation. Step 6 is to integrate the solution sets obtained by the three strategies into the $P_{\text {archive }}$. Then these are sorted using nondominated sort and ${ }_{260} N$ solutions are select into a new population $P_{n e w}$. After that, $P_{n e w}$ is optimized 
Algorithm 4 Diversity maintenance on prediction strategy

Require: Pop(current population), genMax(maximum number of generations),

$\tau_{t}$ (frequency of change $), n_{t}$ (severity of changes), N(population size).

Ensure: $P_{\text {new }}$ (updated population).

1: Initialize a population named as $P_{0}$, set time period $\mathrm{t}=0$, set iteration generation genIter $=0$.

2: If there is no environmental change, turn to 8; else copy the current population as $P_{\text {duplicate }}$ and sort the population using nondominated sort. Then, calculate the nondominated solutions and the center $C_{t}$ according to formula (4).

3: For $P_{\text {duplicate }}$, generate the prediction population $P_{\text {prediction }}$ according to Algorithm 1( $\left.P_{\text {duplicate }}\right)$.

4: For $P_{\text {duplicate }}$, get the gradual search population $P_{\text {GraSearch }}$ according to Algorithm 2( $\left.P_{\text {duplicate }}\right)$.

5: For $P_{\text {duplicate }}$, obtain the diversity maintenance population $P_{D M}$ according to Algorithm 3(P $\left.P_{\text {duplicate }}\right)$.

6: Set current archive $P_{\text {archive }}=P_{\text {prediction }} \cup P_{\text {GraSearch }} \cup P_{D M}$ and act boundary detection on $P_{\text {archive }}$.

7: Sort $P_{\text {archive }}$ using nondominated sort then select $N$ individuals and the population is set $P_{\text {new }}$.

8: Optimize population with RM-MEDA [44].

9: If the termination is satisfied, then output the $P_{n e w}$; else set genIter $=$ genIter +1 , go to 2 .

until the stopping criteria are satisfied.

The boundary check in these strategies is applied to check whether the generated solution is within the given boundary of the decision space. The procedure of the boundary check is described in detail as follows:

$$
y_{i}= \begin{cases}x_{i} & \text { if } l_{i}<x_{i}<u_{i} \\ \operatorname{random}\left(l_{i}, 0.5\left(l_{i}+u_{i}\right)\right) & \text { if } x_{i}<l_{i} \\ \operatorname{random}\left(0.5\left(l_{i}+u_{i}\right), u_{i}\right) & \text { if } x_{i}>u_{i}\end{cases}
$$


where $\operatorname{random}(\mathrm{a}, \mathrm{b})$ is a random function and returns a random value between a and $\mathrm{b}, i=1, \ldots, n$. $n$ is the dimension of the test problems' decision space. $l_{i}$ is the minimum boundary of the $i$-th dimension, and $u_{i}$ is the maximum boundary of the $i$-th dimension.

\section{Test instances and performance indicators}

270 algorithms. The POFs, projections of the POSs and mean points of the POSs can be found in Zhou et al. [35]. Table. 1 lists the test instances mentioned.

Table 1: Test instances used in the paper

\begin{tabular}{llc}
\hline Problems Search Space & Objectives,POS,POF & Remarks \\
\hline & $f_{1}(x)=x_{1}, f_{2}(x)=g \cdot h$. & POF is fixed \\
FDA1 $[0,1] \times[-1,1]^{n-1}$ & $g(x)=1+\sum_{i=2}^{n}\left(x_{i}-G(g(t))\right)^{2}, h(x)=1-\sqrt{f_{1} / g}$. & POS changes \\
& $G(t)=\sin (0.5 \pi t), t=\left\lfloor\tau / \tau_{T}\right\rfloor / n_{t}$. & \\
& $P O S(t): 0 \leq x_{1} \leq 1, x_{i}=G(t), i=2, \ldots, n$. & \\
& $P O F(t): 0 \leq f_{1} \leq 1, f_{2}=1-\sqrt{f_{1} .}$ & \\
& $f_{1}(x)=x_{1}, f_{2}(x)=g \cdot h$. & POF changes \\
FDA2 $\quad[0,1] \times[-1,1]^{n-1}$ & $g(x)=1+\sum_{i=2}^{n} x_{i}^{2}$, & POS is fixed \\
& $\left.h(x)=1-\left(\frac{f_{1}}{g}\right)^{\left(H(t)+\sum_{i=n}^{n} / 2+1\right.}\left(x_{i}-H(t)\right)^{2}\right)^{-1}$. & two objectives \\
& $H(t)=0.75+0.7 \sin (0.5 \pi t), t=\left\lfloor\tau / \tau_{T}\right\rfloor / n_{t}$. & \\
& $P O S(t): 0 \leq x_{1} \leq 1, x_{i}=0, i=2, \ldots, n / 2$, & \\
& $x_{j}=-1, j=n / 2+1, \ldots, n$. &
\end{tabular}




\begin{tabular}{|c|c|c|c|}
\hline \multicolumn{4}{|c|}{ Table 1 continued } \\
\hline & & $P O F(t): 0 \leq f_{1} \leq 1, f_{2}=1-f_{1}^{H(t)^{-1}}$. & \\
\hline \multirow{8}{*}{ FDA3 } & & $f_{1}(x)=x_{1}^{F(t)}, f_{2}(x)=g \cdot h$ & POF changes \\
\hline & {$[0,1] \times[-1,1]^{n-1}$} & $g(x)=1+G(t)+\sum_{i=2}^{n}\left(x_{i}-G(t)\right)^{2}$ & POS changes \\
\hline & & $h(x)=1-\sqrt{f_{1} / g}$ & two objectives \\
\hline & & $G(t)=|\sin (0.5 \pi t)|$ & \\
\hline & & $F(t)=10^{2 \sin (0.5 \pi t)}, t=\left\lfloor\tau / \tau_{T}\right\rfloor / n_{t}$. & \\
\hline & & $P O S(t): 0 \leq x_{1} \leq 1, x_{i}=G(t), i=2, \ldots, n$ & \\
\hline & & $P O F(t): 0 \leq f_{1}=x_{1}^{F(t)} \leq 1$ & \\
\hline & & $f_{2}=(1+G(t))\left(1-\sqrt{\frac{f_{1}}{1+G(t)}}\right)$. & \\
\hline \multirow{8}{*}{ FDA4 } & & $f_{1}(x)=(1+g) \cos \left(0.5 \pi x_{2}\right) \cos \left(0.5 \pi x_{1}\right)$. & $\mathrm{POF}$ is fixed \\
\hline & {$[0,1]^{n}$} & $f_{2}(x)=(1+g) \cos \left(0.5 \pi x_{2}\right) \sin \left(0.5 \pi x_{1}\right)$. & POS changes \\
\hline & & $f_{3}(x)=(1+g) \sin \left(0.5 \pi x_{2}\right)$. & three objectives \\
\hline & & $G(x)=\left|\sum_{i=3}^{n}\left(x_{i}-G\right)^{2}\right|$. & \\
\hline & & $G(t)=\sin (0.5 \pi t), t=\left\lfloor\tau / \tau_{T}\right\rfloor / n_{t}$. & \\
\hline & & $P O S(t): 0 \leq x_{1}, x_{2} \leq 1, x_{i}=G(t), i=3, \ldots, n$ & \\
\hline & & $P O F(t): f_{1}=\cos (u) \cos (v), f_{2}=\cos (u) \sin (v)$ & \\
\hline & & $f_{3}=\sin (u), 0 \leq u, v \leq \pi / 2$ & \\
\hline \multirow{5}{*}{ DMOP1 } & & $f_{1}(x)=x_{1}, f_{2}(x)=g \cdot h$. & POF changes \\
\hline & {$[0,1] \times[-1,1]^{n-1}$} & $g(x)=1+9 \sum_{i=2}^{n} x_{i}^{2}, h(x)=1-\left(\frac{f_{1}}{g}\right)^{H(t)}$ & POS is fixed \\
\hline & & $H(t)=1.25+0.75 \sin (0.5 \pi t), t=\left\lfloor\tau / \tau_{T}\right\rfloor / n_{t}$ & two objectives \\
\hline & & $P O S(t): 0 \leq x_{1} \leq 1, x_{i}=0, i=2, \ldots, n$ & \\
\hline & & $P O F(t): 0 \leq f_{1} \leq 1, f_{2}=1-f_{1}{ }^{H(t)}$ & \\
\hline \multirow{7}{*}{ DMOP2 } & & $f_{1}(x)=x_{1}, f_{2}(x)=g \cdot h$. & POF changes \\
\hline & {$[0,1] \times[-1,1]^{n-1}$} & $g(x)=1+\sum_{i=2}^{n}\left(x_{i}-G(t)\right)^{2}$, & POS changes \\
\hline & & $h(x)=1-\left(\frac{f_{1}}{g}\right)^{H(t)}$ & two objectives \\
\hline & & $G(t)=\sin (0.5 \pi t)$ & \\
\hline & & $H(t)=1.25+0.75 \sin (0.5 \pi t), t=\left\lfloor\tau / \tau_{T}\right\rfloor / n_{t}$ & \\
\hline & & $P O S(t): 0 \leq x_{1} \leq 1, x_{i}=G(t), i=2, \ldots, n$ & \\
\hline & & $P O F(t): 0 \leq f_{1} \leq 1, f_{2}=1-f_{1}{ }^{H(t)}$ & \\
\hline \multirow{6}{*}{ DMOP3 } & & $f_{1}\left(x_{r}\right)=x_{r}, f_{2}\left(x \backslash x_{r}\right)=g \cdot h$. & POF is fixed \\
\hline & {$[0,1] \times[-1,1]^{n-}$} & $g(x)=1+\sum_{i=1}^{x \backslash x_{r}}\left(x_{i}-G(t)\right)^{2}, h(x)=1-\sqrt{\frac{f_{1}}{g}}$. & POS changes \\
\hline & & $G(t)=\sin (0.5 \pi t), r=\cup(1,2, \ldots, n)$ & two objectives \\
\hline & & $t=\left\lfloor\tau / \tau_{T}\right\rfloor / n_{t}$ & \\
\hline & & $P O S(t): 0 \leq x_{1} \leq 1, x_{i}=G(t), i=2, \ldots, n$ & \\
\hline & & $P O F(t): 0 \leq f_{1} \leq 1, f_{2}=1-\sqrt{f_{1}}$. & \\
\hline \multirow{8}{*}{ F5 } & & $f_{1}(x)=\left|x_{1}-a\right|^{H(t)}+\sum_{i \in I_{1}} y_{i}^{2}$ & POF changes \\
\hline & {$[0,5]^{n}$} & $f_{2}(x)=\left|x_{1}-a-1\right|^{H(t)}+\sum_{i \in I_{2}} y_{i}^{2}$. & POS changes \\
\hline & & $y_{i}=x_{i}-b-1+\left|x_{1}-a\right|^{H(t)+\frac{i}{n}}$ & two objectives \\
\hline & & $H(t)=1.25+0.75 \sin (\pi t)$ & \\
\hline & & $a=2 \cos (\pi t)+2, b=2 \sin (2 \pi t)+2$ & \\
\hline & & $t=\left\lfloor\tau / \tau_{T}\right\rfloor / n_{t}$ & \\
\hline & & $I_{1}=\{i \mid 1 \leq i \leq n, i \quad$ is $\quad$ odd $\}$ & \\
\hline & & $I_{2}=\{i \mid 1 \leq i \leq n, i \quad$ is even $\}$ & \\
\hline
\end{tabular}




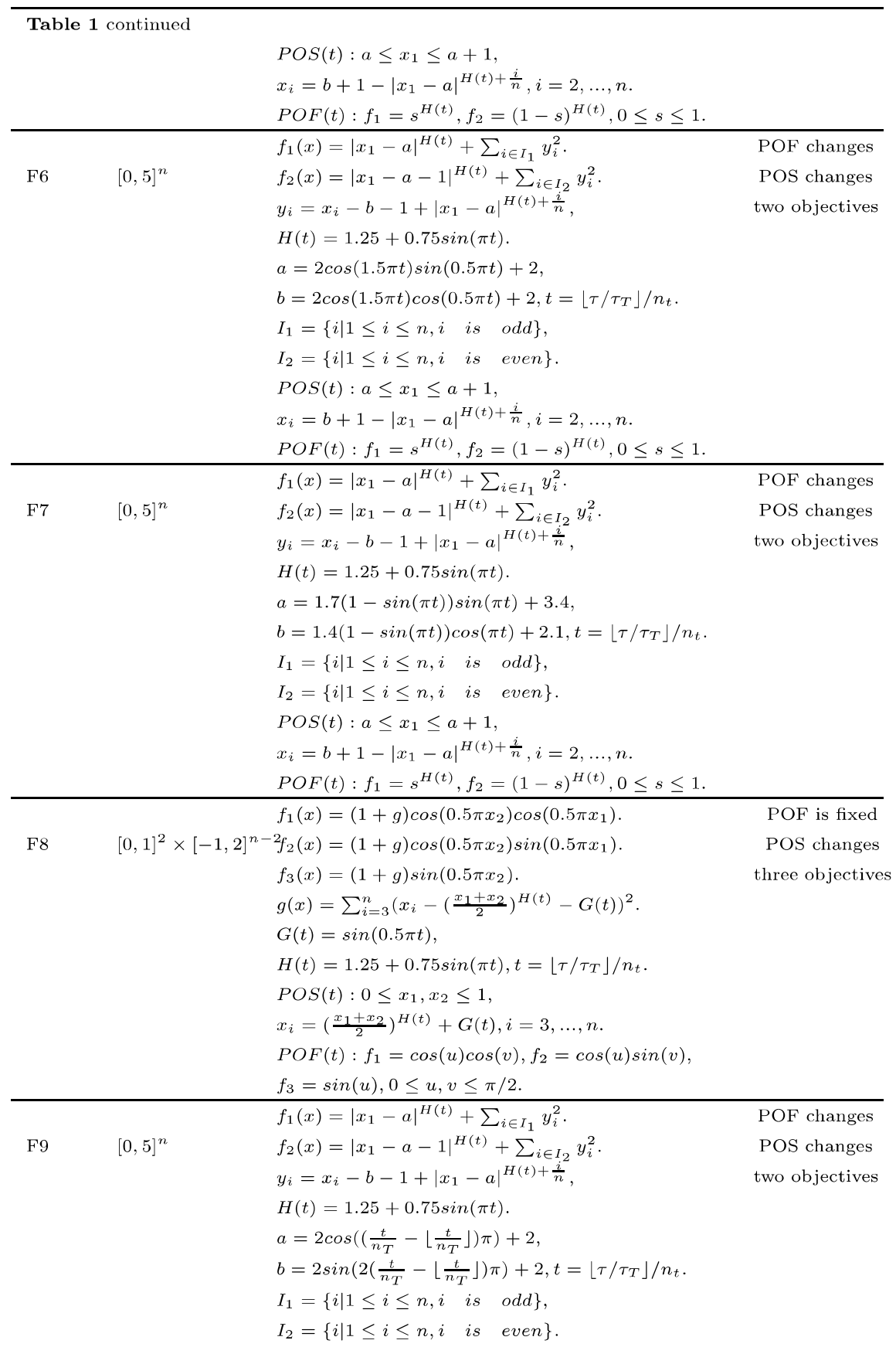


Table 1 continued

$$
\begin{aligned}
& \operatorname{POS}(t): a \leq x_{1} \leq a+1, \\
& x_{i}=b+1-\left|x_{1}-a\right|^{H(t)+\frac{i}{n}}, i=2, \ldots, n . \\
& P O F(t): f_{1}=s^{H(t)}, f_{2}=(1-s)^{H(t)}, 0 \leq s \leq 1 .
\end{aligned}
$$

\subsection{Performance indicators}

285 the algorithms' performance including convergence, distribution and diversity.

(1) Generational Distance (GD): The GD [12] [42] measures the convergence of the obtained solutions by an algorithm. Let $P O F_{t}$ be a set of uniformly distributed Pareto optimal points in the POF at time t, and let $P_{t}$ be the solution obtained by the algorithms. The GD is computed as:

$$
G D\left(P O F_{t}, P_{t}\right)=\frac{\sum_{v \in P_{t}} d\left(P O F_{t}, v\right)}{\left|P_{t}\right|}
$$

where $d\left(P O F_{t}, v\right)=\min _{u \in P O F_{t}} \sqrt{\sum_{j=1}^{m}\left(f_{j}^{u}-f_{j}^{v}\right)^{2}}$ is the minimum Euclidian distance between the point in $P O F_{t}$ and $v$. The lower GD value is, the better convergence the algorithms have.

(2) Schott's Spacing Metric (SP): This kind of indicator was developed by Schott [46] to investigate the distribution of the found Pareto front. The $\mathrm{SP}$ is calculated as follows:

$$
S P=\sqrt{\frac{1}{\left|P_{t}\right|-1} \sum_{i=1}^{\left|P_{t}\right|}\left(D_{i}-\bar{D}\right)^{2}}
$$

where $D_{i}$ is the Euclidean distance between the $i$ th member in $P_{t}$, and its nearest member in $P_{t}$, and $\bar{D}$ is the average value of $D_{i}$. SP measures how evenly the solutions in $\left|P_{t}\right|$ are distributed.

300

(3) Inverted Generational Distance (IGD): The IGD [35] is the comprehensive metric which evaluates the convergence and diversity of the obtained solutions by an algorithm. Let $P O F_{t}$ be a set of uniformly distributed Pareto optimal points in the POF at time $t$, and let $P_{t}$ be the solution obtained by the algorithms. The IGD is computed as:

$$
I G D\left(P O F_{t}, P_{t}\right)=\frac{\sum_{v \in P O F_{t}} d\left(v, P_{t}\right)}{\left|P O F_{t}\right|}
$$


where $d\left(v, P_{t}\right)=\min _{u \in P_{t}} \sqrt{\sum_{j=1}^{m}\left(f_{j}^{v}-f_{j}^{u}\right)^{2}}$ is the minimum Euclidian distance between $v$ and the point in $P_{t}$. The lower IGD value, the better the convergence and distribution of the obtained solution set is.

\section{Experiments}

\subsection{Compared algorithms}

In this section, we use the DMS-MOEA (DMS) which is proposed in this paper. DMS will be compared to three other existing strategies: forward-looking prediction strategy (FPS) [34], population prediction strategy (PPS) [35] and predictive gradient strategy (EGS) [41]. RM-MEDA [44] introduced in Section. 2.2 is chosen as the MOEA optimizer. A brief description of the compared algorithms follows:

(1) FPS: FPS [34] records the boundary points of the POF and predicts the location of the whole population when the next environmental change occurs by autoregressive (AR) model. The re-initialized population in FPS is composed of three parts: the non-dominated solution set, the dominated solution set and the predicted solution set. The function of non-dominated solutions is to let algorithm converge quickly when solving DMOPs whose POS does not change, and the dominated solution set is used to preserve the diversity to search and discover new optimal solutions. The prediction set places a team of individuals in the neighborhood of the next optimum in order to achieve faster discovery and convergence. Due to these features, FPS shows good performance on those problems with fixed POS.

(2) EGS: EGS [41] consists of three parts: mutation, prediction and memory. The mutation operator is carried out on part of the non-dominated solutions in the current population to improve the population's diversity. As for prediction, the evolutionary gradient of the current population is predicted by two parts: the searched final two previous positions of nondominated population center and the last predictive evolutionary gradient. Then some individuals are updated by the predicted gradient and the product of a 
scaling factor. The memory in EGS is based on the center vector $C_{\tau}$ and

variance vector $\widetilde{C_{\tau}} . C_{\tau}$ and $\widetilde{C_{\tau}}$ are calculated by the nondominated solution set after environmental change and then stored in a memory item. Then new individuals are generated according to the normal distribution with the expectation of $C_{\tau}$ and variance of $\widetilde{C_{\tau}}$.

(3) PPS: in PPS [35], the optimal solution set is divided into two parts: the population center and manifold. Based on the archived population centers over a number of continuous time series, PPS adopts a univariate autoregression (AR) model to predict the next population center. Similarly, previous manifolds are used to predict the next manifold. The initial population is completely generated through the predicted center and manifold when there is an environmental change.

\subsection{Parameter settings}

The parameter settings for the instances and algorithms were as follows. The severity and frequency of environmental change were set to be $n_{T}=10$ and $\tau_{T}=30$; the dimensions of the test problems' decision space were $\mathrm{n}=20$. The population size of all test instances was $N=100$. As for change detection, $5 \%$ of the population were randomly selected and reevaluated to detect the environmental change at every generation. Each algorithm ran 20 times for each test problem independently. Then each simulation experiment ran for 3600 generations, and 120 environmental changes were tracked for all strategies.

355 Experimentally, the results of 120 environmental changes were equally divided into 3 stages. Each stage tracked 40 environmental changes, and the mean and standard deviation values of each stage were taken as the results. In Section. 5.4 , the experimental results are shown. Some other key parameter settings in the paper were listed as follows:

- Parameters in DMS: $r_{1}=0.5$ in the prediction strategy, and $m=10$ in the gradual search strategy, and $r_{2}=0.5$ in the random diversity maintenance strategy. 
- Parameters in FPS: The parameters used here were the same as the original FPS. The order in $A R(p)$ model was $p=3$, the number of cluster was set 5 and the length of history mean point series was $M=23$. The probability in the prediction model was 0.9 .

- Parameters in EGS: The number of outdated solutions to retain was 40; the size of the memory archive was 100; the number of individuals selected in prediction was 30; the number of memory items retrieved and the number of evaluations allocated for retrieval were all set as 10 .

- Parameters in PPS: The whole reinitialized population was composed from the predicted individuals. Then the order in the $A R(p)$ model, the number of cluster and the length of history mean point series were equal to the FPS settings values.

\subsection{Computational complexity of the compared strategies and DMS}

The difference of computational complexity in the compared strategies and DMS mainly depends on their own response strategy for the reason that they adopt the same optimization algorithm RM-MEDA. The computational complexity of each strategy is analyzed as follows:

(1) FPS: The mainly computational resource in FPS is spent in the process of prediction using the AR model, and the computational complexity of each point's prediction is $O(n N)$. Then FPS is proposed by predicting the boundary points. Therefore, the computational complexity of EGS is $O(n M N)$ in one generation of the response, where $\mathrm{n}$ is the dimensions of the test problems' decision space, $\mathrm{M}$ is the number of objectives and $\mathrm{N}$ is the population size.

(2) EGS: As introduced in Section. 5.1, the computational complexity of EGS is composed of three parts: prediction, mutation and memory. The prediction operator costs $O\left(M N^{2}\right)$ computations. The mutation and memory all use a truncation operator similar to that used in SPEA2 [47], so the 
two parts spend $O\left(N^{2} \log N\right)$ computations. Therefore, the overall computational complexity of EGS for the response of an environmental change is $O\left(N^{2} \log N\right)$.

(3) PPS: From the original paper of PPS [35], the computational complexity of PPS is composed of the prediction of the center point and manifold. The center point prediction costs $O(n N)$ computational resource and is $O\left(n N^{2}\right)$, and the manifold prediction takes $O\left(n N^{2}\right)$ computational complexity. Therefore, the PPS's computational complexity is $O\left(n N^{2}\right)$.

(4) DMS: The prediction in DMS requires $O(N)$ computational complexity, and the gradual search strategy and the random diversity maintenance strategy all take $O(N)$ computational complexity. Then computational complexity of the overall framework of DMS is mainly spent on the nondominated sort. Therefore, the overall computational complexity of DMS for one response mechanism is $O(N \log N)$.

Based on these analyses, we can conclude that DMS requires less computational complexity than other strategies, indicating DMS is more efficient than other methods in solving DMOPs.

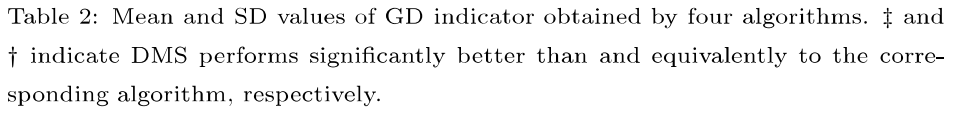

\begin{tabular}{|c|c|c|c|c|c|}
\hline \multicolumn{2}{|c|}{ Problem Strategy } & \multirow{2}{*}{$\begin{array}{c}y \leq t \leq 120 \\
\operatorname{Mean}(\mathrm{SD})\end{array}$} & \multirow{2}{*}{$\begin{array}{l}1 \leq t \leq 40 \\
\operatorname{Mean}(\mathrm{SD})\end{array}$} & \multirow{2}{*}{$\begin{array}{l}41 \leq t \leq 80 \\
\text { Mean(SD) }\end{array}$} & \multirow{2}{*}{$\begin{array}{c}81 \leq t \leq 120 \\
\text { Mean(SD) }\end{array}$} \\
\hline & & & & & \\
\hline \multirow{4}{*}{ FDA1 } & FPS & $0.0292(0.0060) \ddagger$ & $0.0675(0.0180) \ddagger$ & $0.0100(0.0006) \ddagger$ & $0.0100(0.0007) \ddagger$ \\
\hline & EGS & $0.0143(0.0017) \ddagger$ & $0.0243(0.0048) \ddagger$ & $0.0092(0.0009) \ddagger$ & $0.0093(0.0007) \ddagger$ \\
\hline & PPS & $0.0686(0.0295) \ddagger$ & $0.1972(0.0884) \ddagger$ & $0.0044(8.19 \mathrm{E}-05)$ & $0.0043(8.07 E-05)$ \\
\hline & DMS & $0.0069(0.0004)$ & $0.0090(0.0010)$ & $0.0072(0.0005)$ & $0.0058(0.0003)$ \\
\hline \multirow{4}{*}{ FDA2 } & FPS & $0.0136(0.0005)$ & $0.0172(0.0015)$ & $0.0118(0.0002)$ & $0.0119(0.0002)$ \\
\hline & EGS & $0.0131(0.0005)$ & $0.0163(0.0015)$ & $0.0115(0.0003)$ & $0.0114(0.0002)$ \\
\hline & PPS & $0.0139(0.0005) \dagger$ & $0.0174(0.0016)$ & $0.0122(0.0002)$ & $0.0122(0.0002)$ \\
\hline & DMS & $0.0162(0.0005)$ & $0.0182(0.0008)$ & $0.0154(0.0006)$ & $0.0150(0.0009)$ \\
\hline \multirow{4}{*}{ FDA3 } & FPS & $0.1242(0.0040) \ddagger$ & $0.1449(0.0090) \ddagger$ & $0.1156(0.0069) \ddagger$ & $0.1121(0.0069) \ddagger$ \\
\hline & EGS & $0.0782(0.0039) \ddagger$ & $0.0841(0.0085) \ddagger$ & $0.0732(0.0053) \ddagger$ & $0.0772(0.0067) \ddagger$ \\
\hline & PPS & $0.1726(0.0648) \ddagger$ & $0.3628(0.1974) \ddagger$ & $0.0804(0.0165) \ddagger$ & $0.0746(0.0153) \ddagger$ \\
\hline & DMS & $0.0280(0.0008)$ & $0.0290(0.0013)$ & $0.0274(0.0014)$ & $0.0067(0.0009)$ \\
\hline
\end{tabular}




\begin{tabular}{|c|c|c|c|c|c|}
\hline \multicolumn{6}{|c|}{ Table 2 continued } \\
\hline \multirow{4}{*}{ FDA4 } & FPS & $0.2097(0.0099) \ddagger$ & $0.2274(0.0160) \ddagger$ & $0.2033(0.0127) \ddagger$ & $0.1984(0.0131) \ddagger$ \\
\hline & EGS & $0.1616(0.0100) \ddagger$ & $0.1944(0.0190) \ddagger$ & $0.1436(0.0169) \ddagger$ & $0.1467(0.0148) \ddagger$ \\
\hline & PPS & $0.1468(0.0059) \ddagger$ & $0.1841(0.0132) \ddagger$ & $0.1285(0.0063) \ddagger$ & $0.1277(0.0047) \ddagger$ \\
\hline & DMS & $0.1145(0.0035)$ & $0.1309(0.0081)$ & $0.1063(0.0070)$ & $0.1063(0.0053)$ \\
\hline \multirow{4}{*}{ DMOP1 } & FPS & $0.0089(0.0031) \dagger$ & $0.0228(0.0091) \dagger$ & $0.0019(0.0002)$ & $0.0019(0.0004)$ \\
\hline & EGS & $0.0180(0.0070) \ddagger$ & $0.0491(0.0211) \ddagger$ & $0.0024(7.34 \mathrm{E}-05)$ & $0.0024(7.22 \mathrm{E}-05)$ \\
\hline & PPS & $0.0244(0.0677) \ddagger$ & $0.0658(0.2032) \ddagger$ & $0.0037(6.09 \mathrm{E}-05) \ddagger$ & $0.0036(5.61 \mathrm{E}-05) \ddagger$ \\
\hline & DMS & $0.0094(0.0030)$ & $0.0212(0.0091)$ & $0.0035(6.08 \mathrm{E}-05)$ & $0.0035(5.57 \mathrm{E}-05)$ \\
\hline \multirow{4}{*}{ DMOP2 } & FPS & $0.0398(0.0102) \ddagger$ & $0.0962(0.0030) \ddagger$ & $0.0116(0.0011) \ddagger$ & $0.0117(0.0010) \ddagger$ \\
\hline & EGS & $0.0185(0.0018) \ddagger$ & $0.0315(0.0052) \ddagger$ & $0.0120(0.0009) \ddagger$ & $0.0121(0.0010) \ddagger$ \\
\hline & PPS & $0.1149(0.0626) \ddagger$ & $0.3365(0.1877) \ddagger$ & $0.0041(9.82 \mathrm{E}-05)$ & $0.0040(7.69 \mathrm{E}-05)$ \\
\hline & DMS & $0.0078(0.0006)$ & $0.0112(0.0017)$ & $0.0061(0.0004)$ & $0.0059(0.0005)$ \\
\hline \multirow{4}{*}{ DMOP 3} & FPS & $0.0315(0.0067) \ddagger$ & $0.0745(0.0198) \ddagger$ & $0.0100(0.0006) \ddagger$ & $0.0101(0.0008) \ddagger$ \\
\hline & EGS & $0.0144(0.0013) \ddagger$ & $0.0246(0.0038) \ddagger$ & $0.0090(0.0007) \ddagger$ & $0.0094(0.0009) \ddagger$ \\
\hline & PPS & $0.0560(0.0296) \ddagger$ & $0.1595(0.0887) \ddagger$ & $0.0043(9.62 \mathrm{E}-05)$ & $0.0043(0.0001)$ \\
\hline & DMS & $0.0068(0.0003)$ & $0.0087(0.0009)$ & $0.0058(0.0003)$ & $0.0057(0.0004)$ \\
\hline \multirow{4}{*}{ F5 } & FPS & $0.3081(0.2590) \ddagger$ & $0.6697(0.8076) \ddagger$ & $0.1011(0.0453) \ddagger$ & $0.1534(0.1183) \ddagger$ \\
\hline & EGS & $0.0688(0.0161) \ddagger$ & $0.0841(0.0343) \ddagger$ & $0.0612(0.0250) \ddagger$ & $0.0612(0.0225) \ddagger$ \\
\hline & PPS & $0.2610(0.2067) \ddagger$ & $0.7589(0.6186) \ddagger$ & $0.0131(0.0026)$ & $0.0110(0.0006)$ \\
\hline & DMS & $0.0223(0.0060)$ & $0.0292(0.0092)$ & $0.0198(0.0120)$ & $0.0177(0.0062)$ \\
\hline \multirow{4}{*}{$\mathrm{F} 6$} & FPS & $0.4305(0.1548) \ddagger$ & $1.1481(0.4834) \ddagger$ & $0.0720(0.0455) \ddagger$ & $0.0714(0.0720) \ddagger$ \\
\hline & EGS & $0.0761(0.0563) \ddagger$ & $0.1752(0.1713) \ddagger$ & $0.0269(0.0037) \ddagger$ & $0.0262(0.0046) \ddagger$ \\
\hline & PPS & $0.2800(0.0988) \ddagger$ & $0.8190(0.2963) \ddagger$ & $0.0108(0.0007) \ddagger$ & $0.0100(0.0007) \ddagger$ \\
\hline & DMS & $0.0353(0.0247)$ & $0.0876(0.0746)$ & $0.0090(0.0006)$ & $0.0095(0.0017)$ \\
\hline \multirow{4}{*}{ F7 } & FPS & $0.1691(0.0900) \ddagger$ & $0.2870(0.1447) \ddagger$ & $0.0822(0.0627) \ddagger$ & $0.1380(0.2430) \ddagger$ \\
\hline & EGS & $0.0543(0.0450) \ddagger$ & $0.1046(0.1266) \ddagger$ & $0.0301(0.0115) \ddagger$ & $0.0282(0.0053) \ddagger$ \\
\hline & PPS & $0.1275(0.0870) \ddagger$ & $0.3620(0.2610) \ddagger$ & $0.0104(0.0008) \ddagger$ & $0.0100(0.0008) \ddagger$ \\
\hline & DMS & $0.0276(0.0201)$ & $0.0689(0.0602)$ & $0.0069(0.0003)$ & $0.0070(0.0003)$ \\
\hline \multirow{4}{*}{ F8 } & FPS & $0.1955(0.0098) \ddagger$ & $0.2093(0.0189) \ddagger$ & $0.1879(0.0147) \ddagger$ & $0.1894(0.0181) \ddagger$ \\
\hline & EGS & $0.2232(0.0153) \ddagger$ & $0.2676(0.0339) \ddagger$ & $0.2007(0.0236) \neq$ & $0.2014(0.0215) \ddagger$ \\
\hline & PPS & $0.2083(0.0137) \ddagger$ & $0.2632(0.0342) \ddagger$ & $0.1799(0.0143) \ddagger$ & $0.1818(0.0152) \ddagger$ \\
\hline & DMS & $0.1702(0.0084)$ & $0.1852(0.0160)$ & $0.1599(0.0114)$ & $0.1657(0.0149)$ \\
\hline \multirow{4}{*}{ F9 } & FPS & $0.4122(0.2294) \ddagger$ & $0.6683(0.6462) \ddagger$ & $0.2212(0.1570) \ddagger$ & $0.3471(0.2789) \ddagger$ \\
\hline & EGS & $0.0773(0.0251) \ddagger$ & $0.0981(0.0634) \ddagger$ & $0.0684(0.0205) \dagger$ & $0.0654(0.0220) \ddagger$ \\
\hline & PPS & $0.4669(0.2420) \ddagger$ & $1.0434(0.6987) \ddagger$ & $0.2628(0.2585) \ddagger$ & $0.0945(0.0904) \ddagger$ \\
\hline & DMS & $0.0550(0.0150)$ & $0.0486(0.0159)$ & $0.0637(0.0390)$ & $0.0526(0.0288)$ \\
\hline
\end{tabular}

\subsection{Experimental results}

The statistical results including the mean and standard deviation values of

GD, SP and IGD on all instances are shown in Tables. 2, 3 and 4, respectively.

One hundred and twenty environmental changes are divided into three time pe-

riods: $1 \leq t \leq 40,41 \leq t \leq 80,81 \leq t \leq 120$. The mean and standard deviation 
values for $1 \leq t \leq 40,41 \leq t \leq 80,81 \leq t \leq 120$ are presented, respectively, where the best values obtained by one of four algorithms are highlighted in bold face. The Wilcoxon ranksum test [48] was carried out to indicate significance between different results at the 0.05 significance level.

Table 3: Mean and SD of SP indicator obtained by four algorithms. $\ddagger$ and $\dagger$ indicate DMS performs significantly better than and equivalently to the corresponding algorithm, respectively.

\begin{tabular}{|c|c|c|c|c|c|}
\hline \multicolumn{2}{|c|}{ Problem Strategy } & $\begin{array}{c}y \leq t \leq 120 \\
\operatorname{Mean}(\mathrm{SD})\end{array}$ & $\begin{array}{l}1 \leq t \leq 40 \\
\text { Mean(SD) }\end{array}$ & $\begin{array}{l}41 \leq t \leq 80 \\
\text { Mean(SD) }\end{array}$ & $\begin{array}{c}81 \leq t \leq 120 \\
\text { Mean(SD) }\end{array}$ \\
\hline \multirow{4}{*}{ FDA1 } & FPS & $0.0204(0.0239) \dagger$ & $0.0205(0.0238) \dagger$ & $0.0203(0.0239) \dagger$ & $0.0203(0.0239) \dagger$ \\
\hline & EGS & $0.0361(0.0397) \ddagger$ & $0.0361(0.0397) \ddagger$ & $0.0361(0.0398) \ddagger$ & $0.0361(0.0397) \ddagger$ \\
\hline & PPS & $0.0169(0.0154)$ & $0.0178(0.0152)$ & $0.0165(0.0156)$ & $0.0165(0.0156)$ \\
\hline & DMS & $0.0186(0.0151)$ & $0.0186(0.0151)$ & $0.0186(0.0151)$ & $0.0186(0.0151)$ \\
\hline \multirow{4}{*}{ FDA2 } & FPS & $0.0088(0.0018) \dagger$ & $0.0088(0.0018) \dagger$ & $0.0088(0.0018) \dagger$ & $0.0088(0.0018) \dagger$ \\
\hline & EGS & $0.0112(0.0065) \ddagger$ & $0.0113(0.0065) \ddagger$ & $0.0112(0.0065) \ddagger$ & $0.0112(0.0065) \ddagger$ \\
\hline & PPS & $0.0113(0.0054) \ddagger$ & $0.0114(0.0054) \ddagger$ & $0.0113(0.0054) \ddagger$ & $0.0113(0.0054) \ddagger$ \\
\hline & DMS & $0.0087(0.0020)$ & $0.0087(0.0020)$ & $0.0087(0.0020)$ & $0.0087(0.0020)$ \\
\hline \multirow{4}{*}{ FDA3 } & FPS & $0.0161(0.0134) \dagger$ & $0.0161(0.0134) \dagger$ & $0.0161(0.0134) \dagger$ & $0.0161(0.0134) \dagger$ \\
\hline & EGS & $0.0402(0.0425) \ddagger$ & $0.0402(0.0425) \ddagger$ & $0.0402(0.0425) \ddagger$ & $0.0401(0.0425) \ddagger$ \\
\hline & PPS & $0.0145(0.0082)$ & $0.0146(0.0082)$ & $0.0145(0.0081)$ & $0.0144(0.0082)$ \\
\hline & DMS & $0.0156(0.0102)$ & $0.0156(0.0102)$ & $0.0156(0.0102)$ & $0.0156(0.0102)$ \\
\hline \multirow{4}{*}{ FDA4 } & FPS & $0.2776(0.0728) \ddagger$ & $0.2775(0.0729) \ddagger$ & $0.2773(0.0729) \ddagger$ & $0.2781(0.0725) \ddagger$ \\
\hline & EGS & $0.3084(0.0777) \ddagger$ & $0.3067(0.0773) \ddagger$ & $0.3093(0.0779) \ddagger$ & $0.3092(0.0780) \ddagger$ \\
\hline & PPS & $0.2580(0.0680) \ddagger$ & $0.2555(0.0685) \ddagger$ & $0.2593(0.0679) \ddagger$ & $0.2592(0.0678) \ddagger$ \\
\hline & DMS & $0.2123(0.0463)$ & $0.2116(0.0463)$ & $0.2129(0.0463)$ & $0.2125(0.0464)$ \\
\hline \multirow{4}{*}{ DMOP1 } & FPS & $0.0530(0.0436) \dagger$ & $0.0531(0.0436) \dagger$ & $0.0529(0.0436) \dagger$ & $0.0529(0.0436) \dagger$ \\
\hline & EGS & $0.4842(0.7888) \ddagger$ & $0.4842(0.7886) \ddagger$ & $0.4841(0.7889) \ddagger$ & $0.4841(0.7889) \ddagger$ \\
\hline & PPS & $0.0434(0.0357)$ & $0.0435(0.0357)$ & $0.0434(0.0357)$ & $0.0434(0.0357)$ \\
\hline & DMS & $0.0538(0.0844)$ & $0.0538(0.0844)$ & $0.0538(0.0844)$ & $0.0538(0.0844)$ \\
\hline \multirow{4}{*}{ DMOP2 } & FPS & $0.0121(0.0107)$ & $0.0123(0.0107)$ & $0.0120(0.0108)$ & $0.0120(0.0108)$ \\
\hline & EGS & $0.0392(0.0872) \ddagger$ & $0.0391(0.0872) \ddagger$ & $0.0392(0.0872) \ddagger$ & $0.0393(0.0872) \ddagger$ \\
\hline & PPS & $0.0144(0.0157) \dagger$ & $0.0151(0.0156) \dagger$ & $0.0141(0158) \dagger$ & $0.0141(0158) \dagger$ \\
\hline & DMS & $0.0164(0.0118)$ & $0.0164(0.0119)$ & $0.0164(0.0118)$ & $0.0164(0.0118)$ \\
\hline \multirow{4}{*}{ DMOP3 } & FPS & $0.0179(0.0168) \dagger$ & $0.0181(0.0167) \dagger$ & $0.0178(0.0168) \dagger$ & $0.0178(0.0168) \dagger$ \\
\hline & EGS & $0.0641(0.0972) \ddagger$ & $0.0641(0.0972) \ddagger$ & $0.0641(0.0972) \ddagger$ & $0.0641(0.0972) \ddagger$ \\
\hline & PPS & $0.0170(0.0079)$ & $0.0175(0.0075) \dagger$ & $0.0167(0080)$ & $0.0167(0080)$ \\
\hline & DMS & $0.0173(0.0137)$ & $0.0173(0.0137)$ & $0.0173(0.0137)$ & $0.0173(0.0137)$ \\
\hline \multirow{4}{*}{ F5 } & FPS & $0.0347(0.0229) \dagger$ & $0.0397(0.0237) \ddagger$ & $0.0318(0.0242)$ & $0.0328(0.0237)$ \\
\hline & EGS & $0.0432(0.0435) \dagger$ & $0.0430(0.0436) \dagger$ & $0.0432(0.0435) \dagger$ & $0.0432(0.0436) \dagger$ \\
\hline & PPS & $0.0512(0.0496) \ddagger$ & $0.0556(0.0487) \ddagger$ & $0.0490(0.0504) \ddagger$ & $0.0490(0.0504) \ddagger$ \\
\hline & DMS & $0.0339(0.0191)$ & $0.0338(0.0191)$ & $0.0339(0.0191)$ & $0.0339(0.0192)$ \\
\hline
\end{tabular}




\begin{tabular}{|c|c|c|c|c|c|}
\hline \multicolumn{6}{|c|}{ Table 3 continued } \\
\hline \multirow{4}{*}{ F6 } & FPS & $0.0765(0.0805)$ & $0.0794(0.0748)$ & $0.0747(0.0836)$ & $0.0755(0.0834)$ \\
\hline & EGS & $0.1963(0.3021) \ddagger$ & $0.1954(0.3018) \ddagger$ & $0.1968(0.3022) \ddagger$ & $0.1968(0.3022) \ddagger$ \\
\hline & PPS & $0.1425(0.2809) \ddagger$ & $0.1443(0.2794) \ddagger$ & $0.1416(0.2817) \ddagger$ & $0.1416(0.2817) \ddagger$ \\
\hline & DMS & $0.0942(0.1047)$ & $0.0939(0.1044)$ & $0.0944(0.1049)$ & $0.0943(0.1049)$ \\
\hline \multirow{4}{*}{ F7 } & FPS & $0.1015(0.1057) \ddagger$ & $0.1015(0.1053) \ddagger$ & $0.1015(0.1064) \ddagger$ & $0.1014(0.1055) \ddagger$ \\
\hline & EGS & $0.1024(0.1189) \ddagger$ & $0.1019(0.1182) \ddagger$ & $0.1026(0.1191) \ddagger$ & $0.1027(0.1193) \ddagger$ \\
\hline & PPS & $0.0863(0.0944)$ & $0.0863(0.0924)$ & $0.0862(0.0953)$ & $0.0862(0.0953)$ \\
\hline & DMS & $0.0871(0.0716)$ & $0.0868(0.0714)$ & $0.0873(0.0718)$ & $0.0872(0.0713)$ \\
\hline \multirow{4}{*}{ F8 } & FPS & $0.3625(0.1665) \ddagger$ & $0.3622(0.1661) \ddagger$ & $0.3625(0.1669) \ddagger$ & $0.3626(0.1665) \ddagger$ \\
\hline & EGS & $0.6168(0.2575) \ddagger$ & $0.6146(0.2568) \ddagger$ & $0.6181(0.2578) \ddagger$ & $0.6178(0.2580) \ddagger$ \\
\hline & PPS & $0.3442(0.2145) \ddagger$ & $0.3418(0.2143) \ddagger$ & $0.3454(0.2150) \ddagger$ & $0.3454(0.2143) \ddagger$ \\
\hline & DMS & $0.3013(0.1354)$ & $0.3008(0.1353)$ & $0.3017(0.1351)$ & $0.3012(0.1358)$ \\
\hline \multirow{4}{*}{ F9 } & FPS & $0.0429(0.1435) \dagger$ & $0.0464(0.0429) \dagger$ & $0.0407(0.0449) \dagger$ & $0.0417(0.0436) \dagger$ \\
\hline & EGS & $0.0404(0.0194)$ & $0.0402(0.0193)$ & $0.0405(0.0195)$ & $0.0405(0.0195)$ \\
\hline & PPS & $0.0484(0.0518) \dagger$ & $0.0534(0.0517) \dagger$ & $0.0465(0.0517) \dagger$ & $0.0453(0.0525) \dagger$ \\
\hline & DMS & $0.0469(0.0455)$ & $0.0470(0.0455)$ & $0.0469(0.0455)$ & $0.0469(0.0455)$ \\
\hline
\end{tabular}

\subsubsection{Results on FDAs and DMOPs}

The proposed method in this paper (DMS) and the other three methods [34] [35] [41] are tested on FDA [2] and DMOP [12] with linear correlation between decision variables. It can be observed from Table. 2 that DMS gains the best results on the majority of the tested FDA and DMOP problems, indicating that DMS has better convergence over the three other compared strategies in most case. When $0 \leq t \leq 120$ and $1 \leq t \leq 40$, DMS significantly performs better than FPS, PPS, and EGS on all FDAs and DMOPs problems except FDA2. However, when $41 \leq t \leq 80$ and $81 \leq t \leq 120$, DMS surpasses three other strategies on FDA3 and FDA4. For FDA1, DMOP2 and DMOP3, DMS is a little worse than PPS and better than FPS and EGS. On DMOP1 when $41 \leq t \leq 80$ and $81 \leq t \leq 120$, DMS significantly outperforms PPS, but is not better than FPS and EGS.

Table 4: Mean and SD of IGD indicator obtained by four algorithms. $\ddagger$ and $\dagger$ indicate DMS performs significantly better than and equivalently to the corresponding algorithm, respectively.

\begin{tabular}{|c|c|c|c|c|}
\hline \multirow[t]{2}{*}{ Problem Strategy } & $0 \leq t \leq 120$ & $1 \leq t \leq 40$ & $41 \leq t \leq 80$ & $81 \leq t \leq 120$ \\
\hline & $\operatorname{Mean}(\mathrm{SD})$ & $\operatorname{Mean}(\mathrm{SD})$ & $\operatorname{Mean}(\mathrm{SD})$ & Mean(SD) \\
\hline
\end{tabular}




\begin{tabular}{|c|c|c|c|c|c|}
\hline \multicolumn{6}{|c|}{ Table 4 continued } \\
\hline \multirow{4}{*}{ FDA1 } & FPS & $0.0241(0.0043) \ddagger$ & $0.0521(0.0128) \ddagger$ & $0.0101(0.0004) \ddagger$ & $0.0100(0.0005) \ddagger$ \\
\hline & EGS & $0.0131(0.0012) \ddagger$ & $0.0205(0.0035) \ddagger$ & $0.0094(0.0006) \ddagger$ & $0.0094(0.0006) \ddagger$ \\
\hline & PPS & $0.0477(0.0192) \ddagger$ & $0.1314(0.0575) \ddagger$ & $0.0059(6.23 \mathrm{E}-05)$ & $0.0058(6.37 \mathrm{E}-05)$ \\
\hline & DMS & $0.0079(0.0004)$ & $0.0096(0.0009)$ & $0.0072(0.0005)$ & $0.0071(0.0003)$ \\
\hline \multirow{4}{*}{ FDA2 } & FPS & $0.0063(0.0002)$ & $0.0078(0.0006)$ & $0.0056(5.74 \mathrm{E}-05)$ & $0.0056(4.50 \mathrm{E}-05)$ \\
\hline & EGS & $0.0061(0.0002)$ & $0.0075(0.0005)$ & $0.0054(2.90 \mathrm{E}-05)$ & $0.0054(4.30 \mathrm{E}-03)$ \\
\hline & PPS & $0.0066(0.0002)$ & $0.0081(0.0007)$ & $0.0059(3.27 \mathrm{E}-05)$ & $0.0059(2.59 \mathrm{E}-05)$ \\
\hline & DMS & $0.0081(0.0002)$ & $0.0090(0.0003)$ & $0.0078(0.0003)$ & $0.0076(0.0003)$ \\
\hline \multirow{4}{*}{ FDA3 } & FPS & $0.0223(0.0026) \ddagger$ & $0.0296(0.0040) \ddagger$ & $0.0195(0.0040) \ddagger$ & $0.0178(0.0032) \ddagger$ \\
\hline & EGS & $0.0132(0.0018) \ddagger$ & $0.0175(0.0036) \ddagger$ & $0.0105(0.0025) \ddagger$ & $0.0115(0.0027) \ddagger$ \\
\hline & PPS & $0.1030(0.0588) \ddagger$ & $0.2479(0.1785) \ddagger$ & $0.0330(0.0090) \ddagger$ & $0.0279(0.0060) \ddagger$ \\
\hline & DMS & $0.0073(0.0006)$ & $0.0080(0.0007)$ & $0.0071(0.0014)$ & $0.0067(0.0009)$ \\
\hline \multirow{4}{*}{ FDA4 } & FPS & $0.1327(0.0035) \ddagger$ & $0.1450(0.0068) \ddagger$ & $0.1275(0.0037) \ddagger$ & $0.1256(0.0032) \ddagger$ \\
\hline & EGS & $0.1127(0.0029) \ddagger$ & $0.1280(0.0060) \ddagger$ & $0.1048(0.0038) \dagger$ & $0.1053(0.0034) \dagger$ \\
\hline & PPS & $0.1242(0.0027) \ddagger$ & $0.1409(0.0064) \ddagger$ & $0.1156(0.0028) \ddagger$ & $0.1159(0.0026) \ddagger$ \\
\hline & DMS & $0.1080(0.0016)$ & $0.1156(0.0042)$ & $0.1041(0.0025)$ & $0.1043(0.0018)$ \\
\hline \multirow{4}{*}{ DMOP1 } & FPS & $0.0121(0.0056) \dagger$ & $0.0277(0.0168) \dagger$ & $0.0043(8.01 \mathrm{E}-05)$ & $0.0043(6.59 \mathrm{E}-05)$ \\
\hline & EGS & $0.0184(0.0044) \ddagger$ & $0.0460(0.0132) \ddagger$ & $0.0047(4.87 \mathrm{E}-05)$ & $0.0046(4.55 \mathrm{E}-05)$ \\
\hline & PPS & $0.0248(0.0583) \dagger$ & $0.0634(0.1750) \dagger$ & $0.0055(3.25 \mathrm{E}-05) \ddagger$ & $0.0055(3.60 \mathrm{E}-05) \ddagger$ \\
\hline & DMS & $0.0129(0.0043)$ & $0.0281(0.0129)$ & $0.0054(3.85 \mathrm{E}-05)$ & $0.0054(4.23 \mathrm{E}-05)$ \\
\hline \multirow{4}{*}{ DMOP2 } & FPS & $0.0377(0.0090) \ddagger$ & $0.0902(0.0266) \ddagger$ & $0.0114(0.0008) \ddagger$ & $0.0116(0.0008) \ddagger$ \\
\hline & EGS & $0.0172(0.0020) \ddagger$ & $0.0294(0.0056) \ddagger$ & $0.0113(0.0009) \ddagger$ & $0.0111(0.0008) \ddagger$ \\
\hline & PPS & $0.1141(0.0628) \ddagger$ & $0.3308(0.1883) \ddagger$ & $0.0058(6.92 \mathrm{E}-05)$ & $0.0057(5.74 \mathrm{E}-05)$ \\
\hline & DMS & $0.0091(0.0008)$ & $0.0129(0.0022)$ & $0.0071(0.0003)$ & $0.0071(0.0003)$ \\
\hline \multirow{4}{*}{ DMOP3 } & FPS & $0.0261(0.0049) \ddagger$ & $0.0582(0.0146) \ddagger$ & $0.0101(0.0005) \ddagger$ & $0.0101(0.0007) \ddagger$ \\
\hline & EGS & $0.0132(0.0011) \ddagger$ & $0.0210(0.0032) \ddagger$ & $0.0093(0.0005) \ddagger$ & $0.0095(0.0008) \ddagger$ \\
\hline & PPS & $0.0405(0.0191) \ddagger$ & $0.1097(0.0572) \ddagger$ & $0.0058(8.07 \mathrm{E}-05)$ & $0.0059(8.45 E-05)$ \\
\hline & DMS & $0.0078(0.0002)$ & $0.0094(0.0007)$ & $0.0070(0.0002)$ & $0.0070(0.0003)$ \\
\hline \multirow{4}{*}{ F5 } & FPS & $0.0949(0.0514) \ddagger$ & $0.1980(0.1579) \ddagger$ & $0.0409(0.0101) \ddagger$ & $0.0458(0.0130) \ddagger$ \\
\hline & EGS & $0.0510(0.0071) \ddagger$ & $0.0587(0.0115) \ddagger$ & $0.0474(0.0180) \ddagger$ & $0.0470(0.0089) \ddagger$ \\
\hline & PPS & $0.1060(0.0765) \ddagger$ & $0.2936(0.2283) \ddagger$ & $0.0128(0.0013)$ & $0.0115(0.0005)$ \\
\hline & DMS & $0.0150(0.0014)$ & $0.0180(0.0025)$ & $0.0138(0.0025)$ & $0.0133(0.0016)$ \\
\hline \multirow{4}{*}{ F6 } & FPS & $0.1259(0.0277) \ddagger$ & $0.3261(0.0838) \ddagger$ & $0.0261(0.0044) \ddagger$ & $0.0255(0.0063) \ddagger$ \\
\hline & EGS & $0.0478(0.0137) \ddagger$ & $0.0842(0.0405) \ddagger$ & $0.0301(0.0050) \ddagger$ & $0.0291(0.0041) \ddagger$ \\
\hline & PPS & $0.1200(0.0280) \ddagger$ & $0.3383(0.0836) \ddagger$ & $0.0112(0.0008) \ddagger$ & $0.0110(0.0006) \ddagger$ \\
\hline & DMS & $0.0235(0.0049)$ & $0.0516(0.0149)$ & $0.0094(0.0004)$ & $0.0096(0.0004)$ \\
\hline \multirow{4}{*}{ F7 } & FPS & $0.0555(0.0131) \ddagger$ & $0.0989(0.0311) \ddagger$ & $0.0320(0.0104) \ddagger$ & $0.0357(0.0240) \ddagger$ \\
\hline & EGS & $0.0347(0.0056) \ddagger$ & $0.0483(0.0141) \ddagger$ & $0.0279(0.0060) \ddagger$ & $0.0279(0.0046) \ddagger$ \\
\hline & PPS & $0.0524(0.0240) \ddagger$ & $0.1356(0.0719) \ddagger$ & $0.0109(0.0006) \ddagger$ & $0.0107(0.0006) \ddagger$ \\
\hline & DMS & $0.0166(0.0019)$ & $0.0337(0.0056)$ & $0.0081(0.0002)$ & $0.0082(0.0002)$ \\
\hline \multirow{3}{*}{ F8 } & FPS & $0.1256(0.0022) \ddagger$ & $0.1308(0.0040) \ddagger$ & $0.1225(0.0031) \ddagger$ & $0.1234(0.0038) \ddagger$ \\
\hline & EGS & $0.1313(0.0037) \ddagger$ & $0.1438(0.0079) \ddagger$ & $0.1254(0.0055) \ddagger$ & $0.1246(0.0053) \ddagger$ \\
\hline & PPS & $0.1329(0.0037) \ddagger$ & $0.1512(0.0098) \ddagger$ & $0.1240(0.0030) \ddagger$ & $0.1236(0.0028) \ddagger$ \\
\hline
\end{tabular}




\begin{tabular}{|c|c|c|c|c|c|}
\hline \multicolumn{6}{|c|}{ Table 4 continued } \\
\hline & DMS & $0.1194(0.0014)$ & $0.1252(0.0031)$ & $0.1161(0.0017)$ & $0.1169(0.0027)$ \\
\hline \multirow{4}{*}{ F9 } & FPS & $0.1369(0.0646) \ddagger$ & $0.2062(0.1621) \ddagger$ & $0.0884(0.0561) \ddagger$ & $0.1161(0.0890) \ddagger$ \\
\hline & EGS & $0.0652(0.0060) \ddagger$ & $0.0742(0.0198) \ddagger$ & $0.0623(0.0089) \ddagger$ & $0.0591(0.0086) \ddagger$ \\
\hline & PPS & $0.1963(0.0894) \ddagger$ & $0.4039(0.2567) \ddagger$ & $0.1298(0.1119) \ddagger$ & $0.0553(0.0389) \ddagger$ \\
\hline & DMS & $0.0330(0.0061)$ & $0.0306(0.0090)$ & $0.0367(0.0144)$ & $0.0317(0.0115)$ \\
\hline
\end{tabular}

As shown in Table. 3, DMS significantly performs better than EGS on all FDAs and DMOPs in terms of SP. For FPS, DMS significantly surpasses it on FDA4 and DMS shows equal distribution to FPS on FDA1, FDA2, FDA3, DMOP1 and DMOP3, but DMS is a little worse than FPS on DMOP2. When compared to PPS, DMS significantly outperforms it on FDA2 and FDA4, and DMS performs equivalently to PPS on DMOP2. However, DMS shows a little worse performance than PPS on other instances.

It is clear from Table. 4 that when $0 \leq t \leq 120$ and $1 \leq t \leq 40$, DMS performs better than FPS, PPS, and EGS on all FDAs and DMOPs problems except FDA2 and DMOP1. EGS shows better performance than the other three strategies on FDA2. DMS is better than PPS, and worse than FPS and EGS on FDA2. For DMOP1, FPS shows the best performance among the four compared strategies. DMS is a little worse than FPS, and better than EGS and PPS. However, when $41 \leq t \leq 80$ and $81 \leq t \leq 120$, DMS performs better than FPS, PPS, and EGS on FDA3 and FDA4. For FDA1, DMOP2 and DMOP3, DMS performs worse than PPS but surpasses others. On FDA2 and DMOP1 when $41 \leq t \leq 80$ and $81 \leq t \leq 120$, DMS performs better than PPS, but not better than FPS and EGS.

At the beginning of the stage when $1 \leq t \leq 40$ on most instances except FDA2 and DMOP1, DMS performs better than FPS, PPS and EGS in terms of GD and IGD metrics for several reasons. On the one hand, FPS and PPS need the accumulation of history information. Furthermore, the predictive gradient strategy in EGS is not accurate at the first stage. On the other hand, the exploitation in DMS could make up for the inaccuracy of exploration so as to guide the population evolve to the POS. Specifically, DMS adopts the grad- 
ual search strategy to find more good solutions with convergence. Thus, the proposed DMS can respond to environmental changes quickly.

At the later stage of the optimization when $41 \leq t \leq 80$ and $81 \leq t \leq 120$, the POSs of the problems are fixed. FPS and EGS both perform better than others, and DMS is better than PPS. The reason is that FPS reuses a large number of solutions from the old generation when an environmental change is detected. The new obtained population can approximate to the POF very quickly once the POS of DMOP1 is found. Similarly, the gradient prediction strategy in EGS can help the population find the fixed POS, so EGS performs well on FDA2. A conclusion can be made that FPS and EGS are suitable for ${ }_{465}$ these problems with fixed POS. For the problems with changed POS, DMS is better than the other three strategies on FDA3 and FDA4, and a little worse than PPS on FDA1, DMOP2 and DMOP3. FPS can not easily find the new Pareto optimal set by reusing the solutions from the old generation. PPS can perform better than the other three strategies for a reason that it adopts a maturer prediction model to predict the whole population. For DMS, the gradual search strategy in DMS can greatly contribute to making the population find more solutions around the POS. Moreover, the random diversity maintenance strategy in DMS is designed to increase the diversity of the population. In other words, the diversity maintenance mechanism in DMS could make up for the inaccuracy of the prediction.

For the SP metric, each algorithm shows equal performance in every stage, and the results on the SP metric are quite divergent. The reason for the results may be as follows: four algorithms all selected the RM-MEDA as the optimization algorithm, which may have led to a similar distribution on them. Additionally, PPS shows good performance probably for the reason that PPS predicts and generates the whole distributed population to some extend. The initial population in FPS is generated between several predicted boundary points. 


\subsubsection{Results on F5-F9}

F5-F9, which have nonlinear correlation between decision variables, are considered. The statistical results of GD, SP and IGD including mean and standard deviation on F5-F9 over 20 runs are presented in Table. 2, Table. 3 and Table 4. We can see from Table. 2 and Table. 4 that the DMS significantly performs best on F5-F9 when $1 \leq t \leq 40$ and $1 \leq t \leq 120$, and on F6-F9 when $41 \leq t \leq 80$ and $81 \leq t \leq 120$, indicating the best convergence and comprehen-

490 sive performance on DMS. The experimental results demonstrate that DMS has great advantage in solving such problems with a nonlinear correlation between decision variables. The main reason is that for FPS, only a small proportion of the new population is predicted and most solutions are inherited from the previous population in FPS. The gradient prediction strategy and memory strategy in EGS contribute little to the convergence when the problems are complex like F5 to F9. However, DMS mainly utilizes the exploration and the gradual search strategy to enhance the convergence. Meanwhile, the random diversity maintenance strategy can contribute to diversity, and it also enhances convergence to some extent.

Especially on F9, it is obvious from the results in Table. 2 and Table. 4 that DMS is better than FPS, EGS and PPS in every stage, which shows that DMS has the stronger ability to respond to huge environmental changes.

As shown in Table. 3, DMS significantly performs better than PPS on F5, F6 and F8 while DMS is a little worse than PPS on F7. When compared to EGS, DMS significantly surpasses it on F6, F7 and F8 while the SP value of DMS on F5 is a little larger than it. As for FPS, DMS has significant distribution than it on F7 and F8 while the SP metric of DMS is dominated by FPS on F6. For F9, DMS shows equal performance with the other three strategies.

\subsection{Comparison of the distribution of the final obtained population}

510 In order to clearly analyze the performance of the final populations obtained at every stage, five typical test problems including FDA1, DMOP2, F6, F7 and F9 are chosen. The distribution of final populations gained by four strategies 

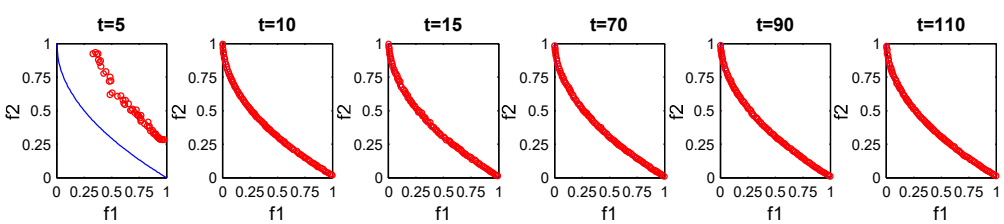

(a)FPS
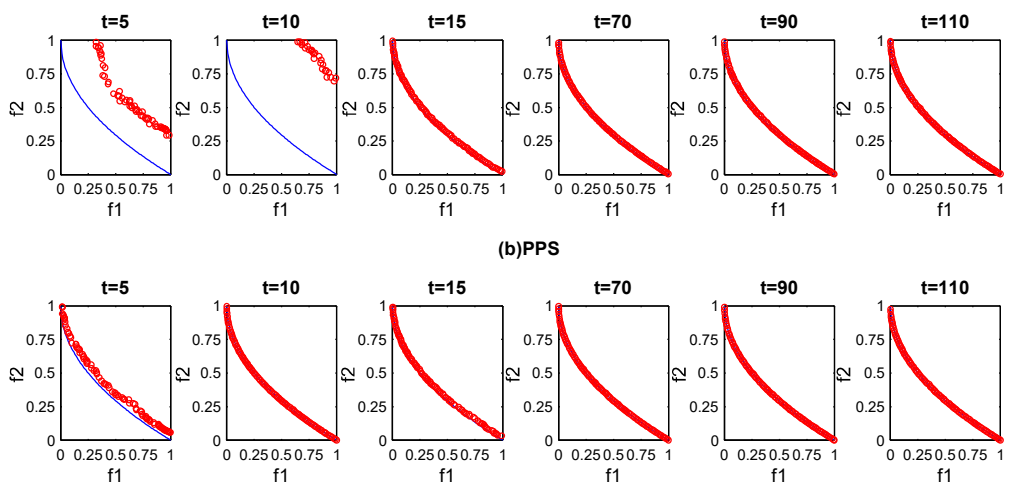

(b)PPS
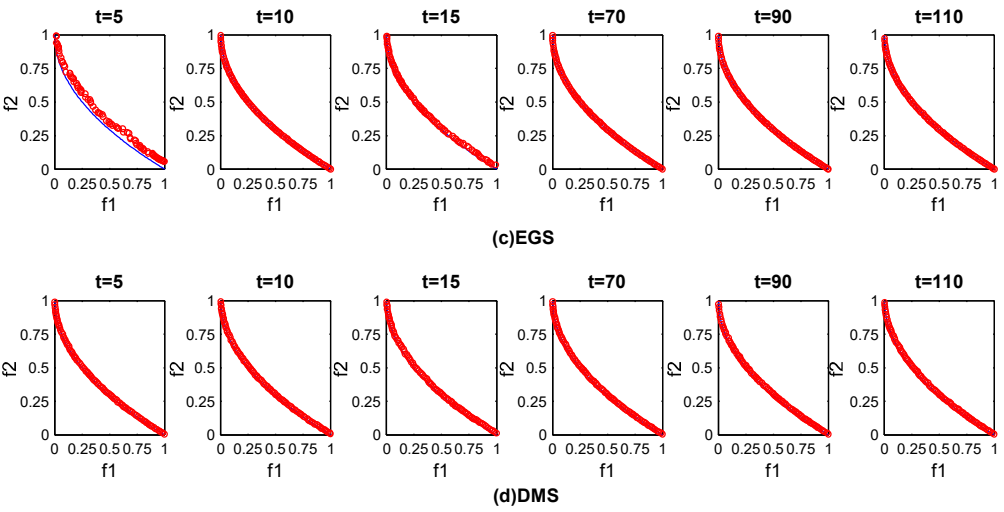

(c)EGS
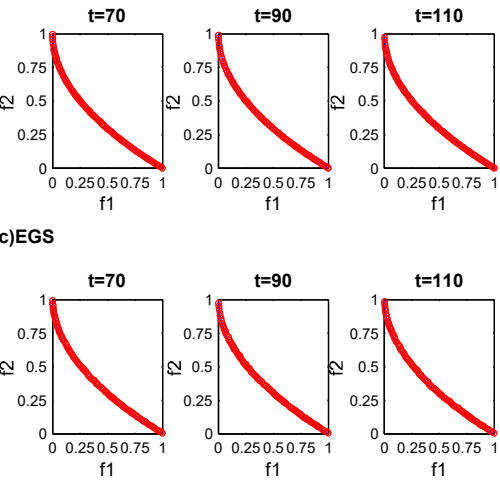

Figure 5: Solution sets obtained by four strategies at six different time steps on FDA1

at different time steps are shown in Figs. 5, 6, 7, 8, 9.

From the Figs. 5, 6, 7, 8, 9, it is obvious that DMS performs better than the other three strategies at the early time steps, which shows that DMS is able to respond to environmental changes more accurately and quickly. FPS performs better than PPS, for the reason that the prediction of PPS needs more history message accumulation than FPS. EGS shows better performance than FPS and PPS due to its gradient prediction strategy and memory strategy. DMS has similar performance on convergence and distribution as PPS in the later time steps. It is also obvious that DMS is better than PPS on the nonliner test 

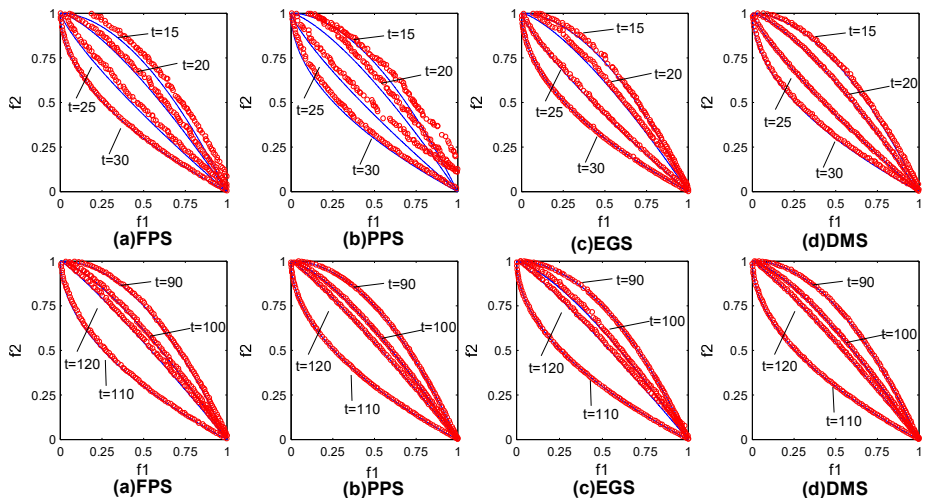

Figure 6: Solution sets obtained by four strategies at eight different time steps on DMOP2
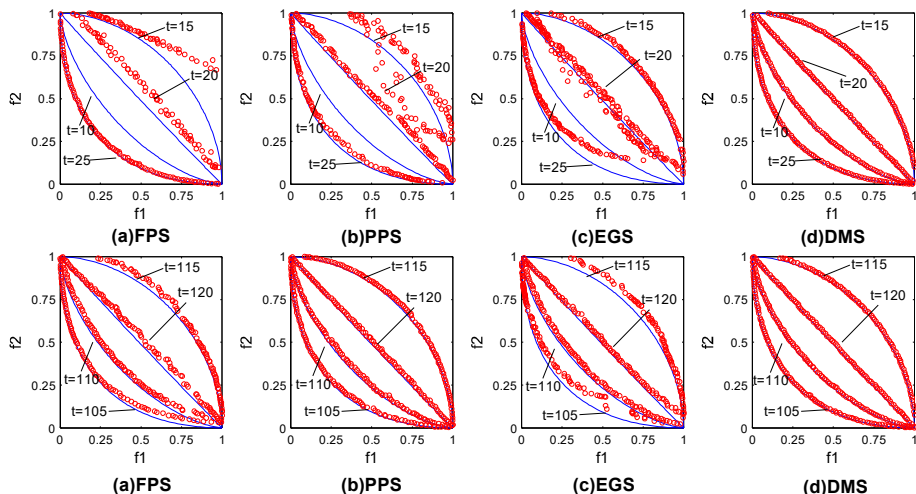

(d)DMS

Figure 7: Solution sets obtained by four strategies at eight different time steps on F6

problems. When solving a complicated problem like F9, DMS has an advantage over the three other strategies. From Fig. 9, it can be seen that the three strategies (FPS, PPS, EGS) have poor convergence and distribution in the all period of environmental changes. DMS is able to make the population converge rapidly and accurately to the Pareto optimal set, and the solutions obtained by DMS are well-distributed and approximating to the POF. Thus, in dealing with complicated nonlinear problems, it has been demonstrated that DMS is competitive against the others. 

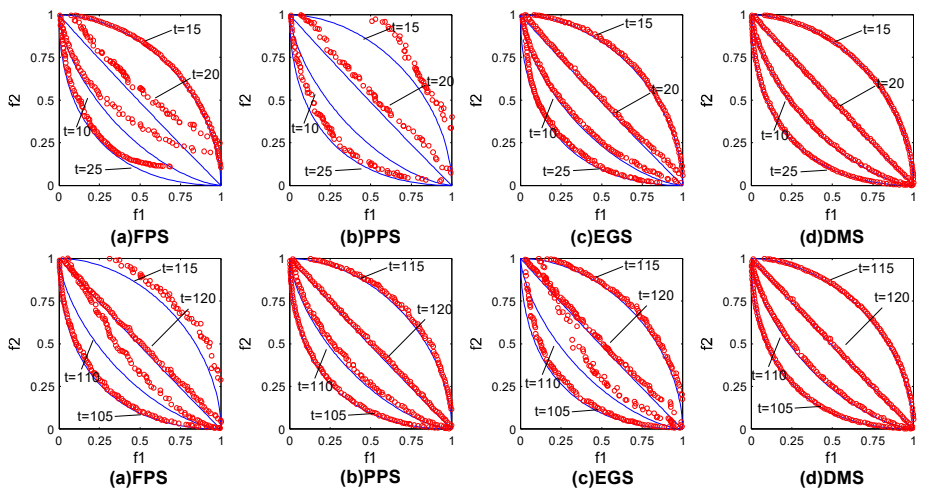

Figure 8: Solution sets obtained by four strategies at eight different time steps on F7

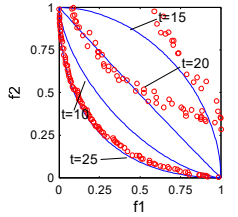

(a)FPS

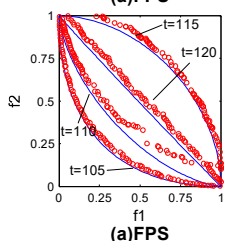

(a)FPS

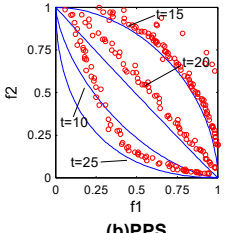

(b)PPS



(b)PPS

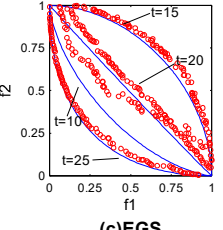

(c)EGS

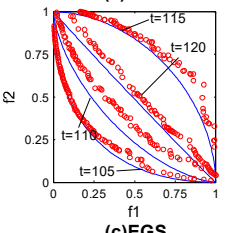

(c)EGS
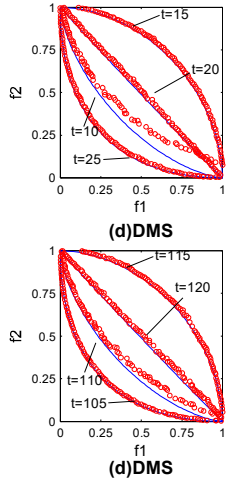

Figure 9: Solution sets obtained by four strategies at eight different time steps on F9

\section{problems}

In order to investigate the influence of the prediction, gradual search, and random diversity maintenance strategies in DMS, several combinations were carried out, and the experiments were conducted on FDA1, FDA3, DMOP2,

F5, F7 and F9. The relevant parameter settings are presented in Section. 5.2. IGD is adopted here for it is a comprehensive performance metric. The obtained average IGD results on three divided stages and their standard deviation values 
are presented in Table .5. The Wilcoxon ranksum test [48] is carried out to indicate significance between different results at the 0.05 significance level.

Table 5: Mean and standard deviation of IGD metric for the algorithms with different strategies on FDA1, FDA3, DMOP2, F5, F7 and F9 over 20 runs. Three strategies: the prediction, gradual search, random diversity maintenance strategies are denoted by $\mathrm{S} 1, \mathrm{~S} 2, \mathrm{~S} 3$ respectively. + shows that $\mathrm{S} 1+\mathrm{S} 2$, $\mathrm{S} 1+\mathrm{S} 3$ and $\mathrm{S} 1+\mathrm{S} 2+\mathrm{S} 3$ significantly surpass $\mathrm{S} 1 . \ddagger$ and $†$ indicate $\mathrm{S} 1+\mathrm{S} 2$ $+\mathrm{S} 3$ performs significantly better than and equivalently to $\mathrm{S} 1+\mathrm{S} 2$ and $\mathrm{S} 1+$ S3, respectively.

\begin{tabular}{|c|c|c|c|c|}
\hline Problem & Strategy & $1 \leq t \leq 40$ & $41 \leq t \leq 80$ & $81 \leq t \leq 120$ \\
\hline & & $\operatorname{Mean}(\mathrm{SD})$ & $\operatorname{Mean}(\mathrm{SD})$ & $\operatorname{Mean}(\mathrm{SD})$ \\
\hline \multirow{4}{*}{ FDA1 } & $\mathrm{S} 1$ & $0.0302(0.0076)+$ & $0.0222(0.0073)+$ & $0.0215(0.0077)+$ \\
\hline & $\mathrm{S} 1+\mathrm{S} 2$ & $0.0122(0.0015) \ddagger$ & $0.0069(0.0003) \dagger$ & $0.0069(0.0002) \dagger$ \\
\hline & $\mathrm{S} 1+\mathrm{S} 3$ & $0.0097(0.0011) \dagger$ & $0.0073(0.0004) \ddagger$ & $0.0072(0.0003) \dagger$ \\
\hline & $\mathrm{S} 1+\mathrm{S} 2+\mathrm{S} 3$ & $0.0096(0.0007)$ & $0.0071(0.0005)$ & $0.0070(0.0002)$ \\
\hline \multirow{4}{*}{ FDA3 } & $\mathrm{S} 1$ & $0.0931(0.0335)+$ & $0.1530(0.0373)+$ & $0.1515(0.0485)+$ \\
\hline & $\mathrm{S} 1+\mathrm{S} 2$ & $0.0117(0.0018) \ddagger$ & $0.0085(0.0023) \dagger$ & $0.0096(0.0030) \ddagger$ \\
\hline & $\mathrm{S} 1+\mathrm{S} 3$ & $0.0089(0.0009) \ddagger$ & $0.0083(0.0023) \ddagger$ & $0.0086(0.0020) \ddagger$ \\
\hline & $\mathrm{S} 1+\mathrm{S} 2+\mathrm{S} 3$ & $0.0080(0.0007)$ & $0.0071(0.0014)$ & $0.0067(0.0009)$ \\
\hline \multirow{4}{*}{ DMOP2 } & $\mathrm{S} 1$ & $0.0518(0.0286)+$ & $0.0334(0.0098)+$ & $0.0325(0.0131)+$ \\
\hline & $\mathrm{S} 1+\mathrm{S} 2$ & $0.0164(0.0037) \ddagger$ & $0.0073(0.0004) \dagger$ & $0.0072(0.0003) \dagger$ \\
\hline & $\mathrm{S} 1+\mathrm{S} 3$ & $0.0140(0.0029) \dagger$ & $0.0074(0.0005) \dagger$ & $0.0071(0.0003) \dagger$ \\
\hline & $\mathrm{S} 1+\mathrm{S} 2+\mathrm{S} 3$ & $0.0129(0.0022)$ & $0.0071(0.0003)$ & $0.0070(0.0003)$ \\
\hline \multirow{4}{*}{ F5 } & S1 & $0.1217(0.0644)+$ & $0.0330(0.0194)+$ & $0.0370(0.0212)+$ \\
\hline & $\mathrm{S} 1+\mathrm{S} 2$ & $0.0616(0.0818) \ddagger$ & $0.0195(0.0090) \ddagger$ & $0.0183(0.0072) \ddagger$ \\
\hline & $\mathrm{S} 1+\mathrm{S} 3$ & $0.0213(0.0056) \ddagger$ & $0.0138(0.0030) \dagger$ & $0.0134(0.0025) \dagger$ \\
\hline & $\mathrm{S} 1+\mathrm{S} 2+\mathrm{S} 3$ & $0.0180(0.0025)$ & $0.0138(0.0025)$ & $0.0133(0.0016)$ \\
\hline \multirow{4}{*}{ F7 } & $\mathrm{S} 1$ & $0.0849(0.0271)+$ & $0.0079(0.0001)+$ & $0.0079(0.0002)+$ \\
\hline & $\mathrm{S} 1+\mathrm{S} 2$ & $0.0429(0.0109) \ddagger$ & $0.0085(0.0002) \ddagger$ & $0.0084(0.0003) \ddagger$ \\
\hline & $\mathrm{S} 1+\mathrm{S} 3$ & $0.0401(0.0099) \ddagger$ & $0.0081(0.0002) \dagger$ & $0.0081(0.0003) \dagger$ \\
\hline & $\mathrm{S} 1+\mathrm{S} 2+\mathrm{S} 3$ & $0.0337(0.0056)$ & $0.0081(0.0002)$ & $0.0081(0.0002)$ \\
\hline \multirow{4}{*}{ F9 } & S1 & $0.2030(0.0789)+$ & $0.2115(0.0882)+$ & $0.2734(0.2147)+$ \\
\hline & $\mathrm{S} 1+\mathrm{S} 2$ & $0.0500(0.0188) \ddagger$ & $0.0570(0.0039) \ddagger$ & $0.0490(0.0199) \ddagger$ \\
\hline & $\mathrm{S} 1+\mathrm{S} 3$ & $0.0448(0.0445) \dagger$ & $0.0611(0.1195) \dagger$ & $0.0388(0.0186) \dagger$ \\
\hline & $\mathrm{S} 1+\mathrm{S} 2+\mathrm{S} 3$ & $0.0306(0.0090)$ & $0.0367(0.0144)$ & $0.0317(0.0115)$ \\
\hline
\end{tabular}


From Table. 5, we can see that combined strategy including both the prediction and gradual search strategies performs significantly better in most problems than the strategy with only prediction. Similarly, the IGD values of the hybrid strategy (prediction and random diversity maintenance strategy) is significantly smaller than that of the strategy with prediction. Moreover, the strategy combining all the strategies (prediction, gradual search strategy and random diversity maintenance strategy) significantly surpasses the single prediction. Thus, from Table. 5, it can be demonstrated that the gradual search and random diversity maintenance strategies contribute to convergence and diversity, which can be seen from the IGD values. The reason is because these two strategies can compensate for the inaccuracy of the prediction during the optimization.

It should be pointed out that the strategy combining three strategies performs significantly better than the strategy consisting of two strategies on the majority of test instances, and the former performs equivalently to the latter on other instances, which indicates that the gradual strategy and the random diversity maintenance strategy have some positive impact on performance when solving problems with different features.

\subsection{Influence from the prediction and diversity maintenance}

The prediction and diversity maintenance strategies (gradual search and random diversity maintenance strategy) play their own role in finding the individuals with good performance. The prediction strategy tries to explore the solutions adjacent to the POS, while the diversity maintenance mechanism exploits the well-distributed solutions in the determined searching decision space. In order to see how much their influence assists the optimization, the average IGD are presented in Fig. 10 on FDA1, DMOP2, F6 and F7 over 20 runs versus 565 time.

From Fig. 10, the RM-MEDA [44] with the prediction strategy performs well according to the low IGD values on FDA1 and DMOP2 when the environmental change is smooth because the problems have linear correlation between the decision variables. While there are some high fluctuations in Fig. 10 (a), and (b), 

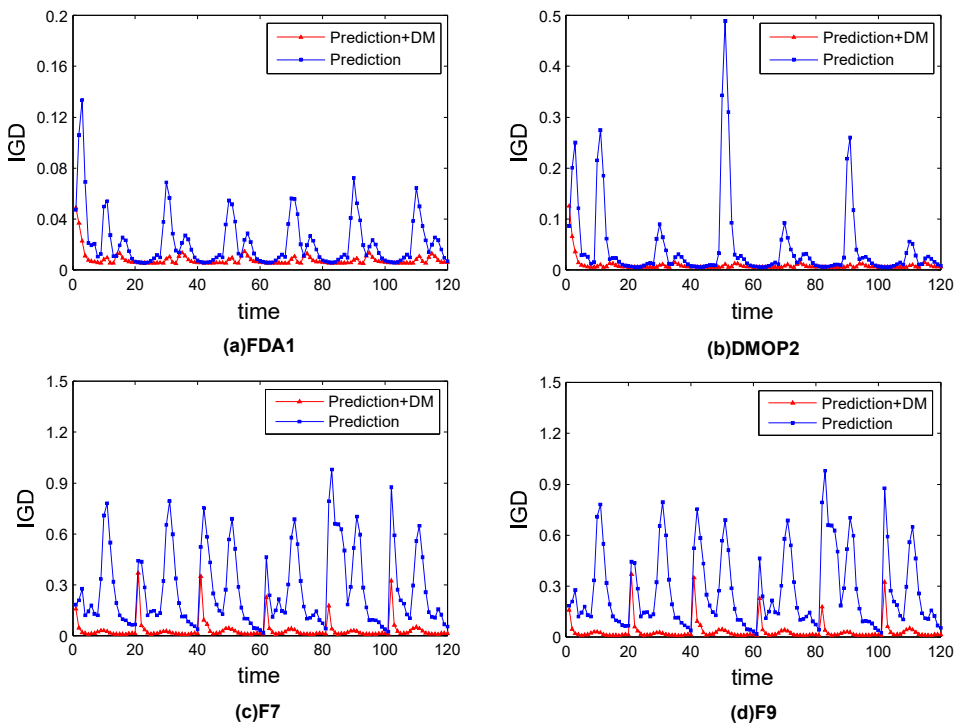

Figure 10: The average IGD over 20 runs versus time on (a) FDA1, (b) DMOP2, (c) F7 and (d) F9, DM represents the combined strategy with gradual search strategy and random diversity maintain strategy.

it may be caused by the sudden environmental changes from the POS. However, for the instances with nonlinear correlation between the decision variables like F7 and F9, the IGD values change frequently and dramatically, as presented in Fig. 10 (c), (d). The reason for that is because the POS is complex and difficult to predict. A conclusion can be made that the prediction can guide the population to evolve towards the POS, but the prediction is not accurate especially in solving complicated problems.

The algorithm with prediction and diversity maintenance strategies has better performance on convergence and distribution than that with the prediction alone. The diversity maintenance strategy will gradually search for more promising individuals, which could prompt the convergence of the population and decrease the inaccuracy that the prediction may lead. Meanwhile, the diversity maintenance mechanism could assist in the improvement of the diversity. Thus, DMS can not only respond to both smooth and sudden environmental changes 
quickly and accurately but also perform well in later environmental changes. In other words, the results demonstrate that the diversity maintenance mechanism has a great promotion to the convergence and diversity in the optimization, and prediction has some influence on optimization to some extent.

\section{Conclusions and future work}

In this paper, to decrease the inaccuracy of predictions, DMS has been proposed for solving DMOPs. The prediction in DMS plays the role of guiding the whole population to evolve to the next period POF. While the prediction may not be accurate, the diversity maintenance mechanism (the gradual search strategy and random diversity maintenance strategy) has been put forward. On the one hand, the gradual search strategy accelerates the speed of converging to the POF, and it also contributes to the diversity to some extent. On the other hand, the random diversity maintenance strategy is being proposed to increase the diversity of the population. Thus, DMS can achieve a good balance between convergence and diversity. Compared with three other dMOEAs, the experiments show that DMS responds faster to environmental changes in problems with linear or nonlinear problems, and also performs better on most of the problems when the environmental change is relatively stable, especially in dealing with complicated nonlinear problems.

Although DMS performs well in dealing with dynamic multi-objective problems, there are some improvements. For instance, DMS could be combined with FPS and PPS to accelerate the convergence due to the good performance of DMS in the early change stages. In addition, there may be other prediction models in machine learning that could be used to predict the POS of DMOPs. Otherwise, it is also an our future work to apply DMS to solve the real engineering problems like dynamic multi-objective optimal control problem [2] and 610 greenhouse control [38]. 


\section{Acknowledgement}

This work was supported by the research projects: the National Natural Science Foundation of China under Grant Nos. 61502408, 61673331, 61379062 and 61403326, the Education Department Major Project of Hunan Province under Grant No. 17A212, the CERNET Innovation Project under Grant No. NGII20150302, the Natural Science Foundation of Hunan Province under Grant No. 14JJ2072, the Science and Technology Plan Project of Hunan Province under Grant No. 2016TP1020, the Provinces and Cities Joint Foundation Project under Grant No. 2017JJ4001.

\section{References}

[1] K. Deb, A. Pratap, S. Agarwal, T. Meyarivan, A fast and elitist multiobjective genetic algorithm: Nsga-ii, Evolutionary Computation, IEEE Transactions on 6 (2) (2002) 182-197.

[2] M. Farina, K. Deb, P. Amato, Dynamic multiobjective optimization problems: test cases, approximations, and applications, Evolutionary Computation, IEEE Transactions on 8 (5) (2004) 425-442.

[3] J. Branke, Evolutionary optimization in dynamic environments, Vol. 3, Springer Science \& Business Media, 2012.

[4] C.-K. Goh, K. C. Tan, Evolutionary multi-objective optimization in uncertain environments, Issues and Algorithms, Studies in Computational Intelligence 186.

[5] J. Y, B. J, Evolutionary optimization in uncertain environments-a survey, Evolutionary Computation, IEEE Transactions on 9 (3) (2009) 303-317.

[6] T. T. Nguyen, S. Yang, J. Branke, Evolutionary dynamic optimization: A survey of the state of the art, Swarm and Evolutionary Computation 6 (2012) 1-24. 
[7] C. A. C. Coello, C. S. P. Zacatenco, 20 years of evolutionary multi-objective optimization: what has been done and what remains to be done, Computational Intelligence: Principles and Practice (2006) 73-88.

[8] S. Yang, M. Li, X. Liu, J. Zheng, A grid-based evolutionary algorithm for many-objective optimization, Evolutionary Computation, IEEE Transactions on 17 (5) (2013) 721-736.

[9] M. Li, S. Yang, X. Liu, Shift-based density estimation for pareto-based algorithms in many-objective optimization, Evolutionary Computation, IEEE Transactions on 18 (3) (2014) 348-365.

[10] R. Liu, W. Zhang, L. Jiao, F. Liu, J. Ma, A sphere-dominance based preference immune-inspired algorithm for dynamic multi-objective optimization, in: Proceedings of the 12th annual conference on Genetic and evolutionary computation, ACM, 2010, pp. 423-430.

[11] Y. Ma, R. Liu, R. Shang, A hybrid dynamic multi-objective immune optimization algorithm using prediction strategy and improved differential evolution crossover operator, in: Neural Information Processing, Springer, 2011, pp. 435-444.

[12] C.-K. Goh, K. C. Tan, A competitive-cooperative coevolutionary paradigm for dynamic multiobjective optimization, Evolutionary Computation, IEEE Transactions on 13 (1) (2009) 103-127.

[13] M. Greeff, A. P. Engelbrecht, Solving dynamic multi-objective problems with vector evaluated particle swarm optimisation, in: Evolutionary Computation, 2008. CEC 2008.(IEEE World Congress on Computational Intelligence). IEEE Congress on, IEEE, 2008, pp. 2917-2924.

[14] J. Wei, Y. Wang, Hyper rectangle search based particle swarm algorithm for dynamic constrained multi-objective optimization problems, in: Evolutionary Computation (CEC), 2012 IEEE Congress on, IEEE, 2012, pp. $1-8$. 
[15] M. R. Sierra, C. A. C. Coello, Improving pso-based multi-objective optimization using crowding, mutation and-dominance, in: Evolutionary MultiCriterion Optimization, Springer, 2005, pp. 505-519.

[16] A. Isaacs, V. Puttige, T. Ray, W. Smith, S. Anavatti, Development of a memetic algorithm for dynamic multi-objective optimization and its applications for online neural network modeling of uavs, in: Neural Networks, 2008. IJCNN 2008.(IEEE World Congress on Computational Intelligence). IEEE International Joint Conference on, IEEE, 2008, pp. 548-554.

[17] R. Shang, L. Jiao, Y. Ren, L. Li, L. Wang, Quantum immune clonal coevolutionary algorithm for dynamic multiobjective optimization, Soft Computing 18 (4) (2014) 743-756.

[18] I. Hatzakis, D. Wallace, Topology of anticipatory populations for evolutionary dynamic multi-objective optimization, in: 11th AIAA/ISSMO Multidisciplinary Analysis and Optimization Conference, 2006, p. 7071.

[19] M. Greeff, E. A. P, Solving dynamic multi-objective problems with vector evaluated particle swarm optimisation, in: Evolutionary Computation (CEC), 2014 IEEE Congress on, IEEE, 2008, pp. 2917-2924.

[20] L. Huang, I. H. Suh, A. Abraham, Dynamic multi-objective optimization based on membrane computing for control of time-varying unstable plants, Information Sciences 181 (11) (2011) 2370-2391.

[21] C. R. Azevedo, A. F. Araújo, Generalized immigration schemes for dynamic evolutionary multiobjective optimization, in: Evolutionary Computation (CEC), 2011 IEEE Congress on, IEEE, 2011, pp. 2033-2040.

[22] A. Díaz-Manríquez, G. T. Pulido, J. G. Ramírez-Torres, Handling dynamic multiobjective problems with particle swarm optimization., in: ICAART (1), 2010, pp. 337-342.

[23] Z. Peng, J. Zheng, J. Zou, A population diversity maintaining strategy based on dynamic environment evolutionary model for dynamic multiob- 
jective optimization, in: Evolutionary Computation (CEC), 2014 IEEE Congress on, IEEE, 2014, pp. 274-281.

[24] S.-U. Guan, Q. Chen, W. Mo, Evolving dynamic multi-objective optimization problems with objective replacement, Artificial Intelligence Review 23 (3) (2005) 267-293.

[25] C.-a. Liu, Y. Wang, New evolutionary algorithm for dynamic multiobjective optimization problems, in: Advances in Natural Computation, Springer, 2006, pp. 889-892.

[26] C.-a. Liu, Y. Wang, A new dynamic multi-objective optimization evolutionary algorithm, International Journal of Innovative Computing Information and Control 4 (8) (2008) 2087-2096.

[27] M. Yang, L. Kang, J. Guan, Multi-algorithm co-evolution strategy for dynamic multi-objective tsp, in: Evolutionary Computation, 2008. CEC 2008.(IEEE World Congress on Computational Intelligence). IEEE Congress on, IEEE, 2008, pp. 466-471.

[28] L. Chun'an, W. Yuping, Multiobjective evolutionary algorithm for dynamic nonlinear constrained optimization problems, Systems Engineering and Electronics, Journal of 20 (1) (2009) 204-210.

[29] Y.-J. Zhang, S.-F. Shao, J. Niyongabo, Cloud hyper mutation particle swarm optimization algorithm based on cloud model, Pattern Recognition and Artificial Intelligence 24 (1) (2011) 90-94.

[30] A. Zhou, Y. Jin, Q. Zhang, B. Sendhoff, E. Tsang, Prediction-based population re-initialization for evolutionary dynamic multi-objective optimization, in: Evolutionary Multi-Criterion Optimization, Springer, 2007, pp. $832-846$.

[31] J. Wei, M. Zhang, Simplex model based evolutionary algorithm for dynamic multi-objective optimization, in: AI 2011: Advances in Artificial Intelligence, Springer, 2011, pp. 372-381. 
[32] E. Vinek, P. P. Beran, E. Schikuta, A dynamic multi-objective optimization framework for selecting distributed deployments in a heterogeneous environment, Procedia Computer Science 4 (2011) 166-175.

[33] M. Liu, W. Zeng, Memory enhanced dynamic multi-objective evolutionary

[35] A. Zhou, Y. Jin, Q. Zhang, A population prediction strategy for evolutionary dynamic multiobjective optimization, Cybernetics, IEEE Transactions on 44 (1) (2014) 40-53.

[36] A. Muruganantham, Y. Zhao, S. B. Gee, Dynamic multiobjective optimiza-

[38] Z. Zhang, Multiobjective optimization immune algorithm in dynamic environments and its application to greenhouse control, Applied Soft Computing 8 (2) (2008) 959-971.

[39] Y. Wang, B. Li, Multi-strategy ensemble evolutionary algorithm for dynamic multi-objective optimization, Memetic Computing 2 (1) (2010) 3-24.

[40] M. Helbig, A. P. Engelbrecht, Archive management for dynamic multiobjective optimisation problems using vector evaluated particle swarm op- 
timisation, in: Evolutionary Computation (CEC), 2011 IEEE Congress on, IEEE, 2011, pp. 2047-2054.

[41] W. T. Koo, C. K. Goh, K. C. Tan, A predictive gradient strategy for multiobjective evolutionary algorithms in a fast changing environment, Memetic Computing 2 (2) (2010) 87-110.

[42] Z. Peng, J. Zheng, J. Zou, M. Liu, Novel prediction and memory strategies for dynamic multiobjective optimization, Soft Computing 19 (9) (2015) 2633-2653.

[43] A. Zhou, Estimation of distribution algorithms for continuous multiobjective optimization, Ph.D. thesis, University of Essex (2009).

[44] Q. Zhang, A. Zhou, Y. Jin, Rm-meda: A regularity model-based multiobjective estimation of distribution algorithm, Evolutionary Computation, IEEE Transactions on 12 (1) (2008) 41-63.

[45] P. Larrañaga, J. A. Lozano, Estimation of distribution algorithms: A new tool for evolutionary computation, Vol. 2, Springer Science \& Business Media, 2001.

[46] J. R. Schott, Fault tolerant design using single and multicriteria genetic algorithm optimization., Tech. rep., DTIC Document (1995).

[47] E. Ziztler, M. Laumanns, L. Thiele, Spea2: Improving the strength pareto evolutionary algorithm for multiobjective optimization, Evolutionary Methods for Design, Optimization, and Control (2002) 95-100.

[48] F. Wilcoxon, Individual comparisons by ranking methods, Biometrics bulletin 1 (6) (1945) 80-83. 